\title{
SMALL-SCALE COSMOLOGICAL PERTURBATIONS: AN ANALYTIC APPROACH
}

\author{
WAYNe HU ${ }^{1}$ AND NaOSHI SUGIYAMA ${ }^{2}$ \\ Received 1995 December 7; accepted 1996 May 16
}

\begin{abstract}
Through analytic techniques verified by numerical calculations, we establish general relations between the matter and cosmic microwave background (CMB) power spectra and their dependence on parameters on small scales. Fluctuations in the CMB, baryons, cold dark matter (CDM), and neutrinos receive a boost at horizon crossing. Baryon drag on the photons causes alternating acoustic peak heights in the $\mathrm{CMB}$ and is uncovered in its bare form under the photon diffusion scale. Decoupling of the photons at last scattering and of the baryons at the end of the Compton drag epoch freezes the diffusion-damped acoustic oscillations into the CMB and matter power spectra at different scales. We determine the dependence of the respective acoustic amplitudes and damping lengths on fundamental cosmological parameters. The baryonic oscillations, enhanced by the velocity overshoot effect, compete with CDM fluctuations in the present matter power spectrum. We present new exact analytic solutions for the cold dark matter fluctuations in the presence of a growth-inhibiting radiation and baryon background. Combined with the acoustic contributions and baryonic infall into CDM potential wells, this provides a highly accurate analytic form of the small-scale transfer function in the general case.
\end{abstract}

Subject headings: cosmic microwave background - cosmology: theory — dark matter elementary particles - large-scale structure of universe

\section{INTRODUCTION}

Small-scale fluctuations in the cosmic microwave background (CMB) and the matter density provide a unique opportunity to probe structure formation in the universe. As CMB anisotropy experiments reach toward smaller and smaller angles, the region of overlap with large-scale structure measurements will increase dramatically. Since the $\mathrm{CMB}$ and matter power spectra encode information at very different epochs in the formation of structure, comparison of the two will provide a powerful consistency test for competing scenarios. Unfortunately, the simple relation between the two power spectrum at large scales (Sachs \& Wolfe 1967) does not hold at small scales. To establish the general relation, we need to employ a more complete analysis of how gravitational instability affects the matter and CMB together.

In previous papers (Hu \& Sugiyama 1995a, 1995b, hereafter $\mathrm{HSa}, \mathrm{HSb}$ ), we developed a conceptually simple analytic description of CMB anisotropy formation. The central advance over previous analytic works (e.g., Doroshkevich, Zeldovich, \& Sunyaev 1978) involved the influence of gravitational potential wells, established by the decoupled matter, on the acoustic oscillations in the photon-baryon fluid. In this paper, we refine the analysis for scales much smaller than the horizon at last scattering as is relevant for large-scale structure calculations. Furthermore, we extend it to encompass the photon-baryon back-reaction on the evolution of the cold dark matter and gravitational potential as well as baryonic decoupling and evolution. This makes it possible to extract the relation between CMB and matter fluctuations established in the early universe.

Analytic solutions in terms of elementary functions can be constructed in the small-scale limit and serve to illuminate the physical processes involved in a model-independent

\footnotetext{
${ }^{1}$ Institute for Advanced Study, School of Natural Sciences, Princeton, NJ 08540; whu@sns.ias.edu

${ }^{2}$ Department of Physics, Kyoto University, Kyoto 606-01, Japan; sugiyama@tap.scphys.kyoto-u.ac.jp
}

manner. Despite the simplicity of the final results, the study of small-scale fluctuations requires a rather technical exposition of perturbation theory. For this reason, we divide this paper into two components. The main text discusses results drawn from a series of appendices and illustrates the corresponding principles in the familiar context of adiabatic cold dark matter (CDM), adiabatic baryonic dark matter (BDM), and isocurvature BDM scenarios for structure formation.

We begin in $\S 2$ with a discussion of the central approximations employed, i.e., the tight coupling limit for the photons and baryons and the external potential representation for metric fluctuations. Details of these methods and the justification of their use can be found in Appendix A. As established in Appendix B, all fluctuations are given a boost at horizon crossing due to the driving effects of gravitational infall and dilation. Since the gravitational potential subsequently decays, the driving effects disappear well after horizon crossing, leaving fluctuations to evolve in their natural source-free modes. For the photon-baryon system, these are acoustic oscillations whose zero point is displaced by baryon drag. As described in $\$ \S 3$ and 4, these oscillations are frozen into the photon and baryon spectra at last scattering and at the end of the Compton drag epoch, respectively. Since these epochs are not equal, photon diffusion (Silk 1968) sets a different damping scale in the CMB and the baryons. This process is described by the acoustic visibility functions introduced in Appendix C.

The baryonic oscillations may be hidden in CDM models by larger cold dark matter fluctuations. These are discussed in $\S 5$. The baryon and radiation background also suppresses CDM growth before the end of the Compton drag epoch. Appendix D treats this source-free evolution of the CDM component analytically. After the end of the Compton drag epoch, the CDM fluctuations provide potential wells into which the baryons fall. From the combined analysis of CMB, baryon, and CDM fluctuations, we obtain accurate analytic expressions for matter and photon transfer functions in the small-scale limit. This improves 
greatly upon the fitting functions for the matter power spectrum in the literature (Bardeen et al. 1986, hereafter BBKS; Peacock \& Dodds 1994; Sugiyama 1995) in the case of a significant baryon fraction. Our form is constructed out of fits from Appendix E for parameters that depend on the detailed physics of recombination, i.e., the last scattering epoch, Compton drag epoch, photon damping length, and Silk damping length.

\section{ACOUSTIC APPROXIMATION}

Previously we developed an analytic description of acoustic oscillations in the photons and baryons ( $\mathrm{HSa}$; $\mathrm{HSb}$ ). As discussed in more detail in Appendix A, this approach is based on two simplifying assumptions: that a perturbative expansion in the Compton scattering time between photons and electrons can be extended up to recombination and that the gravitational potential may be considered as an external field. Combined, these two simplifications imply that photon pressure resists compression by infall into potential wells and sets up acoustic oscillations in the photon-baryon fluid which then damp by photon diffusion. However, both these approximations would seem to be problematic for small-scale fluctuations. Here the optical depth through a wavelength of the fluctuation at last scattering is small, implying weak rather than tight coupling (see, e.g., Kaiser 1984; Hu \& White 1996). This issue is addressed more fully in Appendix C (below eq. [C3]), where it is shown that only acoustic contributions from the tight coupling regime are visible through the last scattering surface, i.e., those from a time slightly earlier than last scattering when the optical depth to Compton scattering was still high.

On the other hand, the second issue poses a computational problem. Inside the horizon in the radiationdominated epoch, the acoustic oscillations in the photon-baryon density feed back into the evolution of the gravitational potential through the generalized Poisson equation. Hu \& White (1996) take the approach of including the self-gravity effects consistently by separating them from the truly external potentials. For many cases, a simpler approach suffices. By considering the self-gravity of the fluid as contributing to an external potential, we retain the conceptual simplicity of $\mathrm{HSa}$ and $\mathrm{HSb}$. Although a consistent evolution of the potential would seem to require a full numerical solution of the coupled equations, it is in practice not generally necessary. The key point is that the feedback effect on the potentials is not arbitrary. Since photon pressure prevents the growth of photon-baryon fluctuations and collisionless damping eliminates neutrino contributions, the potential decreases to zero after horizon crossing in the radiation-dominated epoch. We shall examine how this affects the acoustic oscillations more carefully in $\$ 3$.

Due to Compton drag from the coupling, the baryons density fluctuations follow the photons as $\dot{\delta}_{b}=\frac{3}{4} \dot{\delta}_{\gamma}=3 \dot{\Theta}_{0}$ or $\delta_{b}=3 \Theta_{0}+S$ and exhibit acoustic oscillations as well. Here overdots represent derivatives with respect to conformal time, $\Theta_{0}$ is the isotropic temperature perturbation in Newtonian gauge, and $S$ is the constant photon-baryon entropy. On the other hand, the cold dark matter, if present, is decoupled from photons and suffers only the gravitational effects of the oscillating radiation. Again, since the potential created by the radiation merely oscillates and damps away after horizon crossing, the CDM density perturbations $\delta_{c}$ experience a kick at horizon crossing only to settle into the pure logarithmically growing mode in the radiation-dominated universe.

\section{PHOTON FLUCTUATIONS}

\subsection{Driving Effects}

Before recombination, the photon-baryon system behaves as a damped, forced oscillator. As discussed in $\S 2$, the self-gravity of the acoustic oscillations contributes to the gravitational force on the oscillator at horizon crossing if the photons or baryons contribute significantly to the density of the universe at that epoch. Even though the detailed behavior at horizon crossing in the radiationdominated epoch is complicated by feedback effects, the end result is extremely simple: fluctuations experience a boost proportional to the potential at horizon crossing. As the first compression of the fluid is halted by photon pressure, the self-gravitational potential decays. This leaves the oscillator in a highly compressed state and enhances the amplitude of the subsequent oscillation. For adiabatic initial conditions, the amplitude of the potential at horizon crossing is essentially the initial curvature fluctuation. For isocurvature initial conditions, it is simply related to the initial entropy perturbations.

We quantify these statements in Appendix B and calculate the exact boost factor (see eqs. [B5]-[B6]). It represents an enhancement in temperature fluctuations of a factor of 3 over its initial value in the adiabatic case or more importantly a factor of $5 /\left[1+(4 / 15) f_{v}\right]$ over the gravitational redshift induced large-scale fluctuations in a scale-invariant model (Sachs \& Wolfe 1967). Here the fraction of the radiation energy density contributed by the neutrinos is $f_{v}=$ $\rho_{v} /\left(\rho_{v}+\rho_{\gamma}\right) \simeq 0.405$ for the standard thermal history with three massless species. Its presence accounts for neutrino anisotropic stress, which provides the relation between the gravitational potential $\Psi$ and the curvature fluctuation $\Phi$ (see eq. [A5]). Note that neutrino temperature fluctuations are boosted by a similar factor (Hu et al. 1995).

Three features are worth emphasizing:

1. Potential decay enhances temperature perturbations.

2. Enhancement only occurs for scales that cross the horizon before equality.

3. It occurs at horizon crossing causing phase-coherent oscillations in the wavenumber $k$.

Together the first two points imply that as a function of $k$, the acoustic amplitude will increase through $k_{\text {eq }}$, the horizon crossing wavenumber at equality, forming a potential envelope. By delaying equality, one moves the transitional regime to larger scales and enhances the lower $k$ oscillations. This explains the increasing prominence of the first few acoustic peaks as the matter content $\Omega_{0} h^{2}$ is lowered or the number of relativistic species raised in adiabatic models. It is interesting to note that the effect of equality on the CMB and matter power spectrum are anticorrelated, providing a powerful consistency test for its redshift $z_{\text {eq }}$. As we shall see in $\S 3.2$, though, a complication arises because high- $k$ oscillations are damped by photon diffusion.

The third point follows because the gravitational driving term is effective at sound horizon crossing $\eta \sim 1 / k c_{s}$, thus mimicking a driving frequency of $\tilde{\omega} \sim k c_{s}$. Here $\eta=\int d t / a$ is the conformal time, and we take $c=1$ throughout. Since the natural frequency of the oscillation is related to the 
sound speed $c_{s}$ as $\omega=k c_{s}$, the two scale in the same manner. Thus, the horizon crossing effect is timed to produce a coherent oscillation in $k$. Specifically, adiabatic and isocurvature initial conditions yield cosine and sine temperature oscillations, respectively.

\subsection{Damping Effects}

The photons and baryons are not in fact perfectly coupled, leading to diffusion damping. The coupling strength is quantified by the Compton optical depth $\tau$. Correspondingly, the mean free path of the photons in the baryons is given by $\dot{\tau}^{-1}$. Explicitly, $\dot{\tau}=x_{e} n_{e} \sigma_{\mathrm{T}} a$, where $x_{e}$ is the electron ionization fraction, $n_{e}$ is the electron number density, and $\sigma_{\mathrm{T}}$ is the Thomson cross section. As the photons random walk across a wavelength of the perturbation, temperature fluctuations damp collisionally. More specifically, diffusion generates viscosity or anisotropic stress in the photon-baryon fluid and causes heat conduction across the wavelength (Weinberg 1972, p. 568). Both these processes damp fluctuations. In 1 order of magnitude, the damping length is the random walk distance $(\eta / \dot{\tau})^{1 / 2}$. It exceeds the wavelength of a fluctuation when the optical depth through a single wavelength $\dot{\tau} / k=k \eta$. If the diffusion scale is well under the horizon, $k \eta \gg 1$, so that $\dot{\tau} / k$ is still high at crossover. The photons and baryons are thus still strongly coupled, and the damping may be calculated under the tight coupling approximation.

A quantitative treatment of diffusion damping is given in Appendix A. Subtle effects such as the angular and polarization dependence of Compton scattering enhance slightly the generation of viscosity and thus damping in the radiationdominated universe (Kaiser 1983). The end result of the calculation is a wavenumber $k_{D}(\eta)$ by which acoustic fluctuations are damped as $\exp \left[-\left(k / k_{D}\right)^{2}\right]$ (see eq. [A14]). Combined with the potential envelope from the horizon crossing boost of $\S 3.1$, this completes the acoustic envelope shown in Figure 1. Remaining after diffusion damping is the acoustic offset of $\Theta_{0}+\Psi=-R \Psi$, where $R=3 \rho_{b} / 4 \rho_{\gamma}=$ $31.5 \Omega_{b} h^{2} \Theta_{2.7}^{-4}\left(z / 10^{3}\right)^{-1}$. Here $\Theta_{2.7}=T_{0} / 2.7 \mathrm{~K}$ is the scaled present temperature of the $\mathrm{CMB}, \Omega_{b}$ is the fraction of critical density contributed by the baryons, and the Hubble constant is $H_{0}=100 \mathrm{hm} \mathrm{s}^{-1} \mathrm{Mpc}^{-1}$. We add the Newtonian potential $\Psi$ to the temperature perturbation $\Theta_{0}$ to remove the effect of gravitational redshift on the photons (see Appendix A). The $R \Psi$ term represents the baryon drag effect on the photons and is analogous to the Silk mechanism (Silk 1968), with the roles of the photons and baryons interchanged. Infalling baryons drag the photons into potential wells, leading to a displacement of the zero point of the acoustic oscillation (HSa). Thus, it is responsible for the alternating peak heights and amplitude enhancement of intermediate scale acoustic oscillations (see Appendix A, Figure $8 a$ ). The zero-point shift remains even after diffusion damping has eliminated the oscillations themselves.

\subsection{Decoupling}

As the total optical depth to the present drops below unity, $\tau\left(z_{*}\right)=1$, last scattering of the CMB photons freezes the acoustic oscillations into the spectrum. The optical depth drops rapidly as neutral hydrogen forms so that last

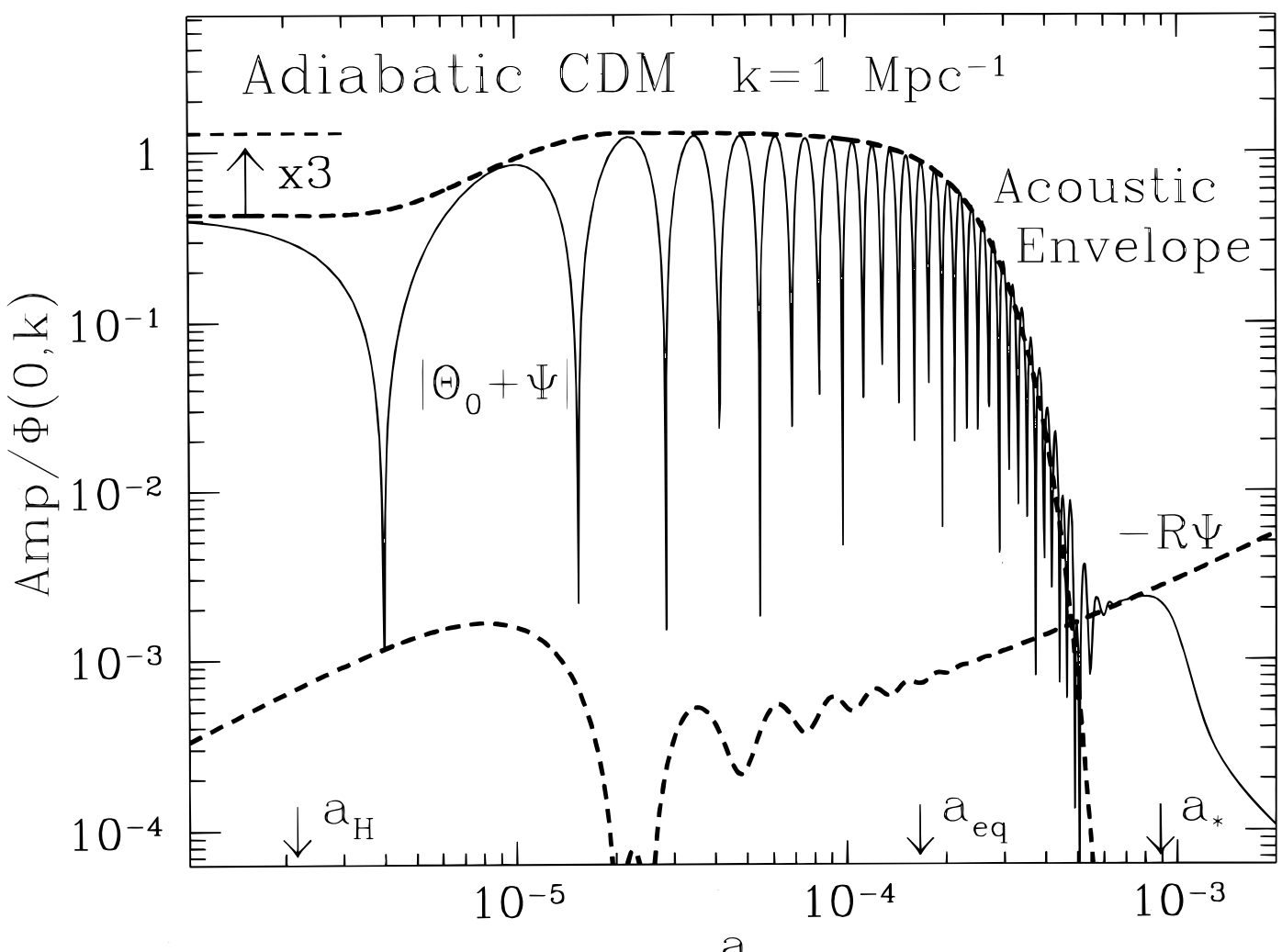

FIG. 1.-Acoustic oscillations receive a boost at horizon crossing $a_{H}$ due to driving from gravitational potential decay. The perturbations then settle into a pure acoustic mode and are subsequently damped by photon diffusion. Together the potential driving and diffusion damping effects form the acoustic envelope. After diffusion damping has destroyed the acoustic oscillations, the underlying baryon drag effect becomes apparent. Since $\Psi$ is constant here for $a \gg a_{\text {eq }},|R \Psi|$ increases with time. This contribution itself is damped at last scattering $a_{*}$ by cancellation. Well after last scattering $a \gg a_{*}$, isotropic perturbations damp collisionlessly, creating angular fluctuations in the CMB. The model here is adiabatic CDM, $\Omega_{0}=1, h=0.5, \Omega_{b}=0.05$. 

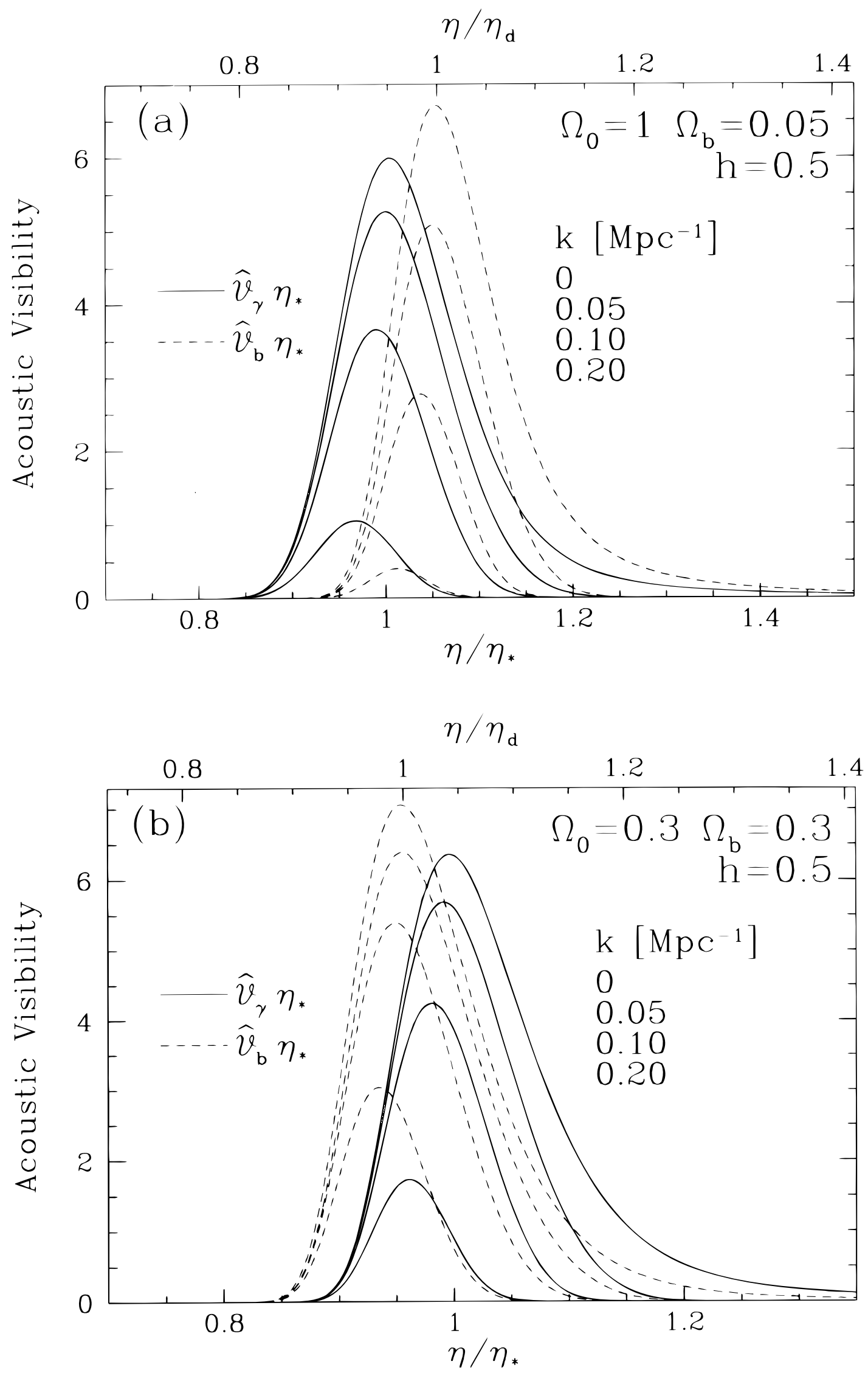

FIG. 2.-Visibility functions for the photons and baryons. In the undamped $k \rightarrow 0$ limit, the photon acoustic visibility and the Compton visibility are equivalent, $\hat{\mathscr{V}}_{\gamma}=\mathscr{V}_{\gamma}$, and the baryon acoustic visibility equals the drag visibility, $\hat{\mathscr{V}}_{b}=\mathscr{V}_{b}$. If $\Omega_{b} h^{2} \lesssim 0.03, \mathscr{V}_{b}$ peaks at later times than $\mathscr{V}_{\nu}$, i.e., $\eta_{d}>\eta_{*}$. For small scales, the acoustic visibilities, which weight the acoustic contributions from the tight coupling regime, are suppressed increasingly at later times by damping, and the width and amplitude of the acoustic visibilities decrease as $k$ increases. 

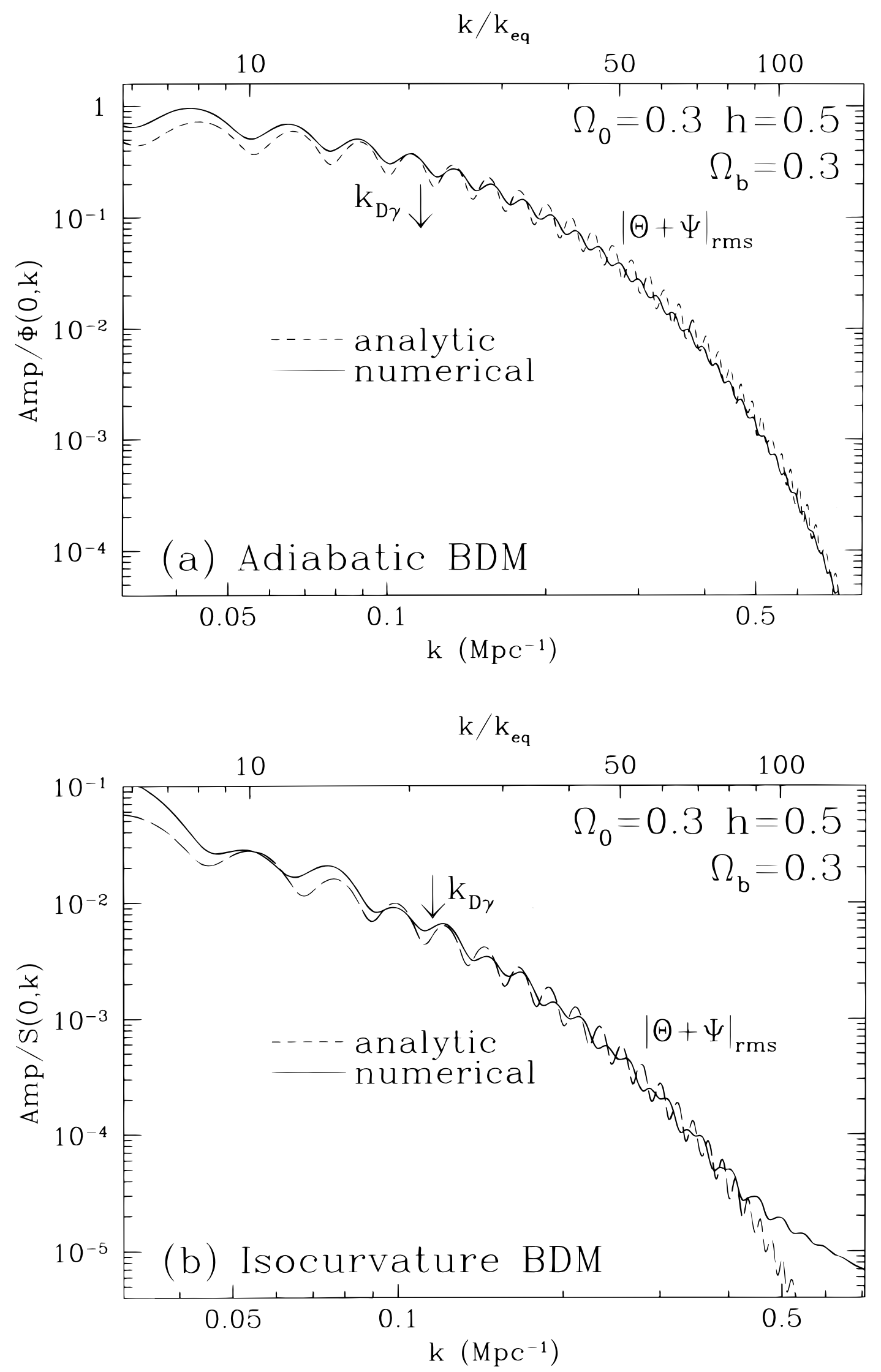

Fig. 3.-Photon transfer function. Plotted is the present rms photon temperature fluctuation relative to the initial curvature fluctuation $\Phi(0, k)$ for adiabatic fluctuations and the initial entropy fluctuation $S(0, k)$ for isocurvature fluctuations assuming standard recombination. The intrinsic amplitude is approximated by the analysis in Appendix B for scales well inside the horizon at equality $k \gg k_{\text {eq }}$. The damping is well approximated by the tight coupling expansion for high $\Omega_{b} h^{2}$ and is overestimated slightly for low values. Below the damping tail, the baryon drag offset clearly appears in $(b)$ and $(c)$, where the gravitational potential is not dominated by acoustic density fluctuations. An analytic treatment of this effect is given in Appendices A and C. 


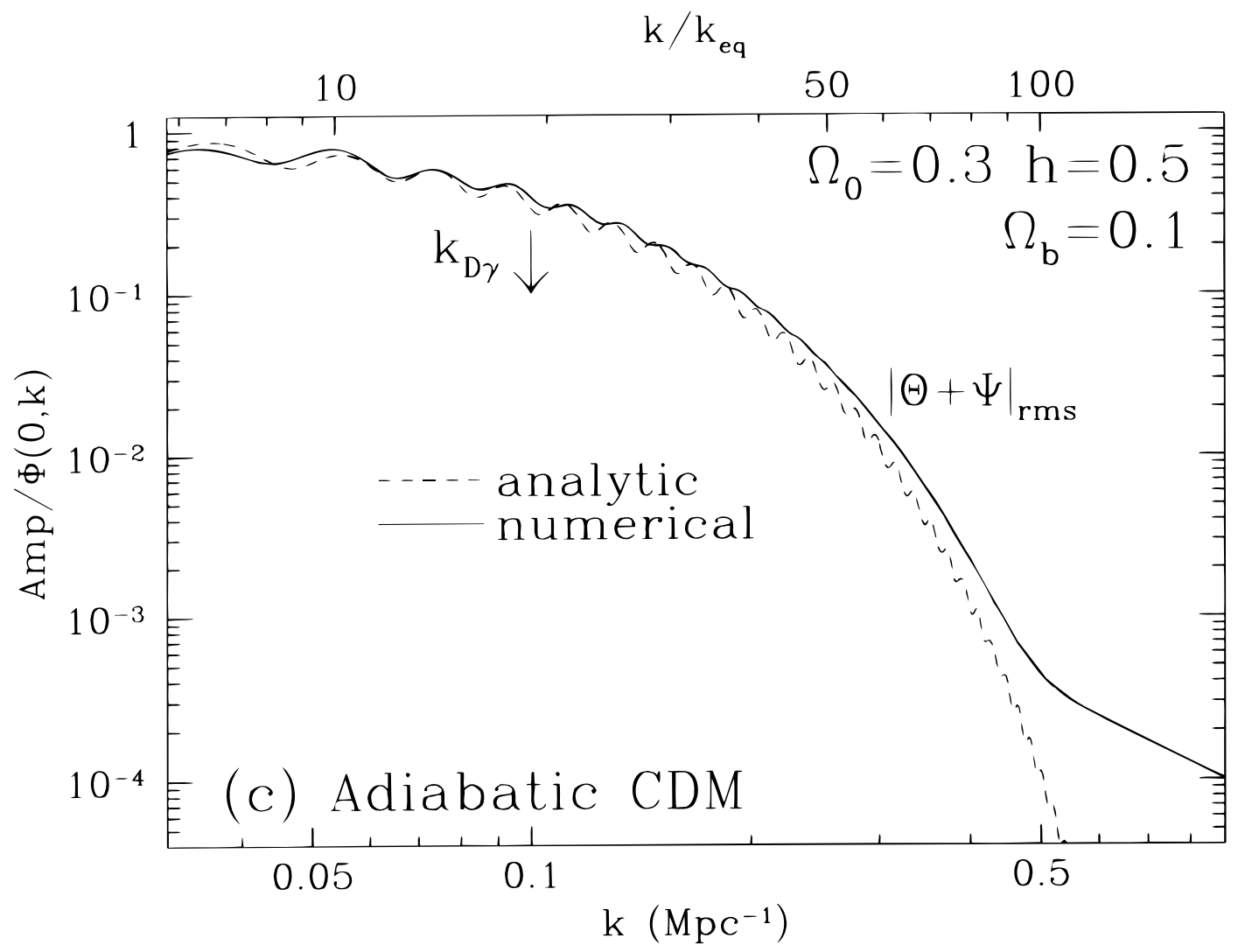

Fig. 3.-Continued

scattering and recombination coincide approximately in the absence of subsequent reionization. This epoch is nearly independent of cosmological parameters such as the matter and baryon content $\Omega_{0} h^{2}$ and $\Omega_{b} h^{2}$ (Peebles 1968; Jones \& Wyse 1985). However, variations at the $10 \%$ level do occur across the full range of parameters. In Appendix E (eq. [E1]), we present an extremely accurate analytic fit to the last scattering epoch.

The phase of the acoustic oscillation is frozen in at the value $k r_{s}\left(\eta_{*}\right)$, where (HSa)

$$
r_{s}=2 \frac{\sqrt{3}}{3}\left(\Omega_{0} H_{0}^{2}\right)^{-1 / 2} \sqrt{\frac{a_{\mathrm{eq}}}{R_{\mathrm{eq}}}} \ln \frac{\sqrt{1+R}+\sqrt{R+R_{\mathrm{eq}}}}{1+\sqrt{R_{\mathrm{eq}}}},
$$

is the sound horizon with $R_{\mathrm{eq}}=R\left(a_{\mathrm{eq}}\right)$ and $a_{\mathrm{eq}}=2.35$ $\times 10^{-5}\left(\Omega_{0} h^{2}\right)^{-1}\left(1-f_{v}\right)^{-1} \Theta_{2.7}^{4}$. Its variation with $k$ produces an oscillatory pattern in the CMB temperature with extrema at scales $(\mathrm{HSb})$

$$
k_{\gamma j} r_{s}\left(\eta_{*}\right)= \begin{cases}j \pi & \text { adiabatic }, \\ \left(j-\frac{1}{2}\right) \pi & \text { isocurvature . }\end{cases}
$$

The physical scale of the peaks $k_{\gamma j}$ is projected onto an angular scale on the sky and provides a sensitive angular diameter distance test for curvature in the universe.

To determine the amplitude of the fluctuations, this instantaneous decoupling approximation must be modified to account for diffusion damping through recombination. The differential visibility function $\mathscr{V}_{\gamma}=\dot{\tau} e^{-\tau}$ expresses the probability that a photon last scattered within $d \eta$ of $\eta$. This function describes how fluctuations at recombination are frozen into the CMB. Equally useful for our purposes is the acoustic visibility function $\hat{\mathscr{V}}_{\gamma}=\mathscr{V}_{\gamma} \exp \left[-\left(k / k_{D}\right)^{2}\right]$. By including the intrinsic damping behavior of the fluctuations, it describes how acoustic oscillations are frozen into the CMB (see Fig. 2). Due to the growth of the diffusion length through recombination, this function is weighted to slightly earlier times than $\mathscr{V}_{\gamma}$. At small scales, only the small fraction of photons which last scattered before $z_{*}$, and hence in the tight coupling regime, retain acoustic fluctuations. This leads to a sharp decrease in acoustic fluctuations with $k$. More specifically, the damping envelope is given by $(\mathrm{HSa})$

$$
\mathscr{D}_{\gamma}(k)=\int_{0}^{\eta_{0}} d \eta \hat{\mathscr{V}}_{\gamma}(\eta, k)=\int_{0}^{\eta_{0}} d \eta \mathscr{V}_{\gamma}(\eta) e^{-\left[k / k_{D}(\eta)\right]^{2}},
$$

i.e., a near exponential damping in $k$. Through the first decade of the drop, it is well approximated by the form

$$
\mathscr{D}_{\gamma}(k) \simeq e^{-\left[k / k_{\gamma}\right]^{m \gamma}} .
$$

The damping angle is given by the same projection conversion as the acoustic peaks $l_{D}=k_{D \gamma} r_{\theta}\left(\eta_{*}\right)$. Accurate fitting functions for $k_{D \gamma}$ and $m_{\gamma}$ are given in Appendix E, equations (E4)-(E7). Note that $k_{D \gamma}$ is the wavenumber at which diffusion suppresses the fluctuation by $e^{-1}$. It is interesting to note that $k_{D y}^{-1}$ is related intimately to the width of the Compton visibility function. This is because the thickness of the last scattering surface is by definition the diffusion length at last scattering. Combining the damping envelope with the intrinsic amplitude of acoustic oscillations discussed in $\S 2$ and Appendix B, we obtain the transfer func- 
tion at small scales. This function represents the growth to the present from the initial curvature or entropy perturbation. In Figure 3, we plot examples for the adiabatic BDM, isocurvature BDM, and adiabatic CDM models with standard recombination. Note that in more typical isocurvature BDM models (Peebles 1987a, 1987b), reionization may occur soon after recombination. In this limit, the damping length continues to grow until it reaches the horizon at the new last scattering surface and destroys all oscillations in the photons.

The corresponding anisotropy can be obtained by choosing an initial curvature and entropy spectrum $\Phi(0, k)$ and $S(0, k)$ and employing the free streaming solution (see $\mathrm{HSa}$, eq. [12]) for the monopole and dipole contributions to the rms given in Appendix B, equation (B7). Note that for low $\Omega_{b} h^{2}$ models, the damping length is somewhat overestimated by the tight coupling approximation. This is not surprising because as $\Omega_{b} h^{2} \rightarrow 0$, the photon mean free path approaches the horizon. In this case, the diffusion length passes the wavelength of the fluctuation when the optical depth through a wavelength is near unity and the tight coupling expansion of Appendix A breaks down. The damping length is overestimated because the photons essentially free stream across the wavelength and do not suffer collisional damping. A phenomenological correction for this effect is given in Appendix E.

In summary, the general features of the CMB fluctuation spectrum are as follows:

1. Acoustic peaks at harmonics of the angle the sound horizon subtends at last scattering, sensitive to $(a)$ the curvature and $(b)$ the adiabatic or isocurvature nature of the initial fluctuations;

2. An acoustic envelope for the oscillation amplitude sensitive to $(a)$ The matter-radiation ratio, e.g., the adiabatic acoustic envelope varies from $(1 / 3) \Psi$ to $(5 / 3) \Psi$ as one passes through the equality scale, $(b)$ The baryon-photon ratio due to the a modulation of $R \Psi$ from baryon drag, and (c) The thermal history from the exponential diffusion-damping tail.

The exact nature of the features will depend on details of the model.

\section{BARYON FLUCTUATIONS}

In the tight coupling limit, the baryons participate in the acoustic oscillations of the photons. Near recombination, they decouple from the photons, though not exactly at last scattering. Scattering represents an exchange of momentum between the two fluids and seeks to equalize their bulk velocities $V_{b}$ and $V_{\gamma}$. However, the two momentum densities $\left(\rho_{\gamma}+p_{\gamma}\right) V_{\gamma}=(4 / 3) \rho_{\gamma} V_{\gamma}$ and $\left(\rho_{b}+p_{b}\right) V_{b} \simeq \rho_{b} V_{b}$ are not equal. Thus, momentum conservation requires that the rate of change of the baryon velocity due to Compton drag is scaled by a factor of $R^{-1}=(4 / 3) \rho_{\gamma} / \rho_{b}$ compared with the photon case; i.e., $\propto \dot{\tau}_{d}=\dot{\tau} / R$. The explicit expression for the baryon momentum conservation or Euler equation is given in Appendix A and is used in Appendix $\mathrm{C}$ to make the qualitative statements here rigorous. The form of the coupling suggests that we can define a drag depth $\tau_{d}\left(\eta_{d}\right)=$ $\int_{\eta_{d}}^{\eta_{0}} \dot{\tau}_{d} d \eta$. Below drag depth $\tau_{d}\left(\eta_{d}\right)=1$, the baryons decouple dynamically from the photons. For the standard recombination scenario, this occurs near recombination but at a different value than last scattering. Analytic fitting formulae for $z_{d}$ are given in Appendix E, equation (E2). Since recombination occurs around $z=10^{3}$ for all models, the end of the drag epoch precedes last scattering if $\Omega_{b} h^{2} \gtrsim 0.03$.

The acoustic fluctuations in the baryons are frozen in at the drag epoch rather than at last scattering. Furthermore, unlike for the CMB, it is not the acoustic density fluctuation that forms peaks in the observable spectrum today but rather the acoustic velocity. This is because the baryon fluctuations continue to evolve. In Appendix $\mathrm{C}$, we give the exact matching conditions at $z_{d}$ onto the growing and decaying modes of pressureless perturbation theory. This yields an accurate description for the subsequent evolution of the baryonic fluctuations in the presence of a background radiation energy density and cold dark matter. Qualitatively, the acoustic velocity at the drag epoch dominates over the acoustic density for the growing mode of fluctuations due to the velocity overshoot effect (Sunyaev \& Zeldovich 1970; Press \& Vishniac 1980). The former moves matter and produces clumping in the baryon density. Since expansion damps peculiar velocities, this lasts for approximately an expansion time $\eta_{d}$. Thus, only scales smaller than the horizon $k \eta_{d} \gg 1$ experience a boost due to the velocity at release. We show an example in Figure 4, where the $k$-mode is chosen to be near a zero point of the acoustic density oscillation at $\eta_{d}$. The rapid regeneration of density fluctuations via velocity overshoot is due to the fact that zeros of the density oscillation are maxima of the velocity oscillation. Therefore, the peaks in the matter power spectrum due to baryonic acoustic oscillations occur at

$$
k_{b j} r_{s}\left(\eta_{d}\right)= \begin{cases}\left(j-\frac{1}{2}\right) \pi & \text { adiabatic, } \\ j \pi & \text { isocurvature, }\end{cases}
$$

and they are roughly $\pi / 2$ out of phase with the corresponding CMB fluctuations.

To obtain the amplitude of the acoustic fluctuations, we must consider damping effects also. Photon diffusion in the tight coupling regime damps baryon fluctuations as well due to Compton drag, i.e., via the Silk mechanism (Silk 1968). Analogous to the photon case, we can construct the drag visibility function $\mathscr{V}_{b}$ out of the drag optical depth $\tau_{d}$. The acoustic visibility function then becomes $\widehat{\mathscr{V}}_{b}=\mathscr{V}_{b}$ exp $\left[-\left(k / k_{D}\right)^{2}\right]$ (see Fig. 2). Similarly, the net damping as a function of scale is described by

$$
\begin{aligned}
\mathscr{D}_{b}(k) & =\int_{0}^{\eta_{0}} d \eta \widehat{\mathscr{V}}_{b}(\eta, k) \\
& =\int_{0}^{\eta_{0}} d \eta \mathscr{V}_{b}(\eta) e^{-\left[k / k_{D}(\eta)\right]^{2}} \simeq e^{-(k / k \mathrm{~s})^{m_{\mathrm{S}}}},
\end{aligned}
$$

where the approximation is valid through the first decade of damping. Analytic fitting formulae for $k_{\mathrm{S}}$ and $m_{\mathrm{S}}$ are given in Appendix E, equations (E9)-(E10). As is the case with the photons, the latter accounts for the width of the visibility function and is almost independent of cosmological parameters. Note that the Silk damping length is not the same as the photon damping length despite the underlying similarity in cause. The small difference between last scattering and the drag epoch can alter it significantly due to the rapid change in $k_{D}$ around recombination (see Fig. 2). Moreover, the two scale differently with the baryon and matter content.

Together with the horizon crossing boost from Appendix $B$, this defines the contribution to the matter transfer func- 


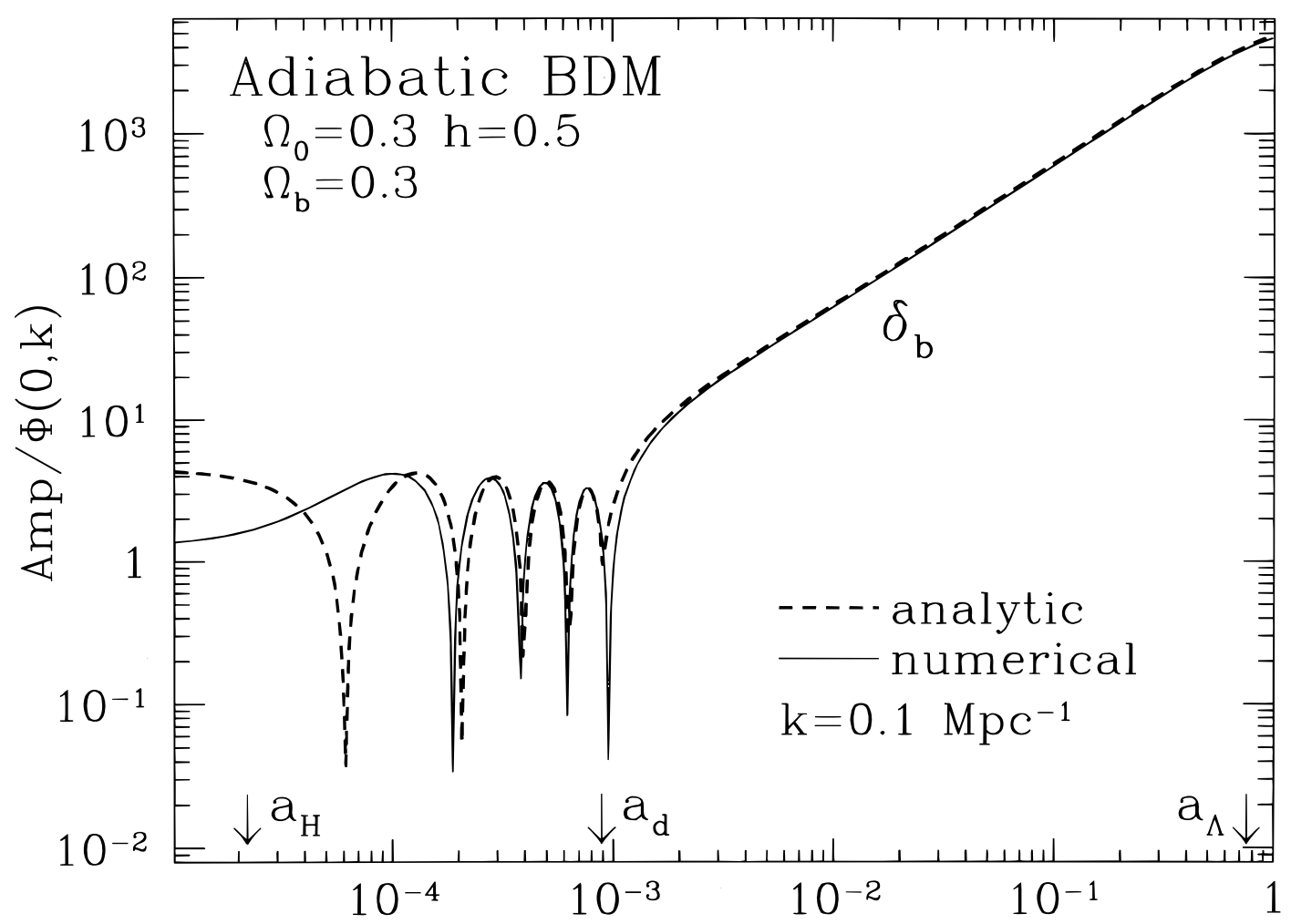

a

FIG. 4.-Baryon fluctuation time evolution. The baryon density fluctuation $\delta_{b}$ follows the photons before the drag epoch $a_{d}$ yielding a simple oscillatory form for $a_{H} \ll a \ll a_{d}$. The Silk damping length is given by the diffusion length at the drag epoch. The portion of the baryon fluctuations that enter the growing mode is dominated by the velocity perturbation at the drag epoch $a_{d}$ due to the velocity overshoot effect (see also Fig. 11). Since a flat $\Lambda$ model is chosen here, the growth rate is slowed by the rapid expansion for $a \gtrsim a_{\Lambda}=\left(\Omega_{0} / \Omega_{\Lambda}\right)^{1 / 3}$.

tion of the acoustic oscillations (see Fig. 5). Explicit expressions for the three scenarios shown are given in Appendix D, equations (D22) and (D23). In the isocurvature BDM models, the transfer function at small scales is dominated by the initial entropy fluctuation $S(0, k)$. Furthermore, unlike the photon oscillations, baryon oscillations may survive early reionization if it occurs more than an expansion time after the drag epoch. In this case, the baryonic oscillations are subsequently surrounded by a homogeneous and isotropic distribution of photons. They then represent entropy perturbations and are not damped by further photon diffusion. Even in the rather unphysical event of nearinstantaneous reionization, baryonic oscillations may survive if reionization is accompanied by the formation of a significant fraction of compact baryonic objects (see, e.g., Gnedin \& Ostriker 1990).

If the model contains cold dark matter, baryons suffer an additional effect. After the drag epoch, they fall into potential wells established by the CDM. If the CDM to baryon ratio is high, this effect will dominate over the velocity overshoot of the acoustic oscillations. To quantify this effect, we need to consider the evolution of CDM fluctuations.

\section{COLD DARK MATTER FLUCTUATIONS}

Let us begin with the evolution of CDM fluctuations before the drag epoch. As shown in $\S 2$ and Appendix B2, CDM fluctuations are given a kick at horizon crossing that sends them into logarithmically growing mode. As the universe becomes matter dominated, this stimulates power-law growing and decaying modes $\delta_{c} \propto a^{p}$. If CDM dominates the nonrelativistic matter, $p=\{1,-(3 / 2)\}$. However, if the baryon fraction is significant, the power law is modified. To first order in $\Omega_{b} / \Omega_{0}$,

$$
p=\left\{1-\frac{3}{5} \frac{\Omega_{b}}{\Omega_{0}}, \quad-\frac{3}{2}\left(1-\frac{2}{5} \frac{\Omega_{b}}{\Omega_{0}}\right)\right\} .
$$

CDM growth is thus inhibited by the presence of baryons. We give the exact solution to the evolution equation from horizon crossing to $z_{d}$ in Appendix D. Because of the complexity of these expressions, it is also useful and instructive to obtain approximate scaling relations. If $a_{d} \gg a_{\mathrm{eq}}$ and $\Omega_{b} / \Omega_{0} \ll 1$, the main effect is an amplitude reduction of the CDM density perturbation $\delta_{c}\left(\eta_{d}, k\right)$ by approximately $\left(a_{d} / a_{\mathrm{eq}}\right)^{-0.6 \Omega_{b} / \Omega_{0}} \simeq\left(24 \Omega_{0} h^{2}\right)^{-0.6 \Omega_{b} / \Omega_{0}}$ (see also Fig. 10). As $\Omega_{0} h^{2}$ is lowered, the drag epoch recedes into the radiation domination epoch and the regime in which the growth rate is affected, $a_{\mathrm{eq}}<a<a_{d}$, vanishes.

At the drag epoch, the baryons are released from the photons and behave dynamically as if they were CDM. Since baryonic infall into CDM wells subsequently contributes to the self-gravity of the matter, the growth rates again become $p=\{1,-(3 / 2)\}$ regardless of the baryon fraction. Thus, we follow the total nonrelativistic matter perturbation $\delta_{m}$ after the drag epoch. The relative contribution of the CDM fluctuations at the drag epoch to the total nonrelativistic matter fluctuations $\delta_{m}$ scales as $1-\Omega_{b} / \Omega_{0}$. A good fit to the net suppression is given by the form

$$
\delta_{m} \simeq \alpha\left(1-\frac{\Omega_{b}}{\Omega_{0}}\right) \lim _{\Omega_{b} \rightarrow 0} \delta_{m},
$$

where $\alpha \simeq\left(46 \Omega_{0} h^{2}\right)^{-0.67 \Omega_{b} / \Omega_{0}}$ for low $\Omega_{b} / \Omega_{0} \lesssim 0.5$ and high $\Omega_{0} h^{2} \gg 0.03$. The change in the coefficients from the naive 

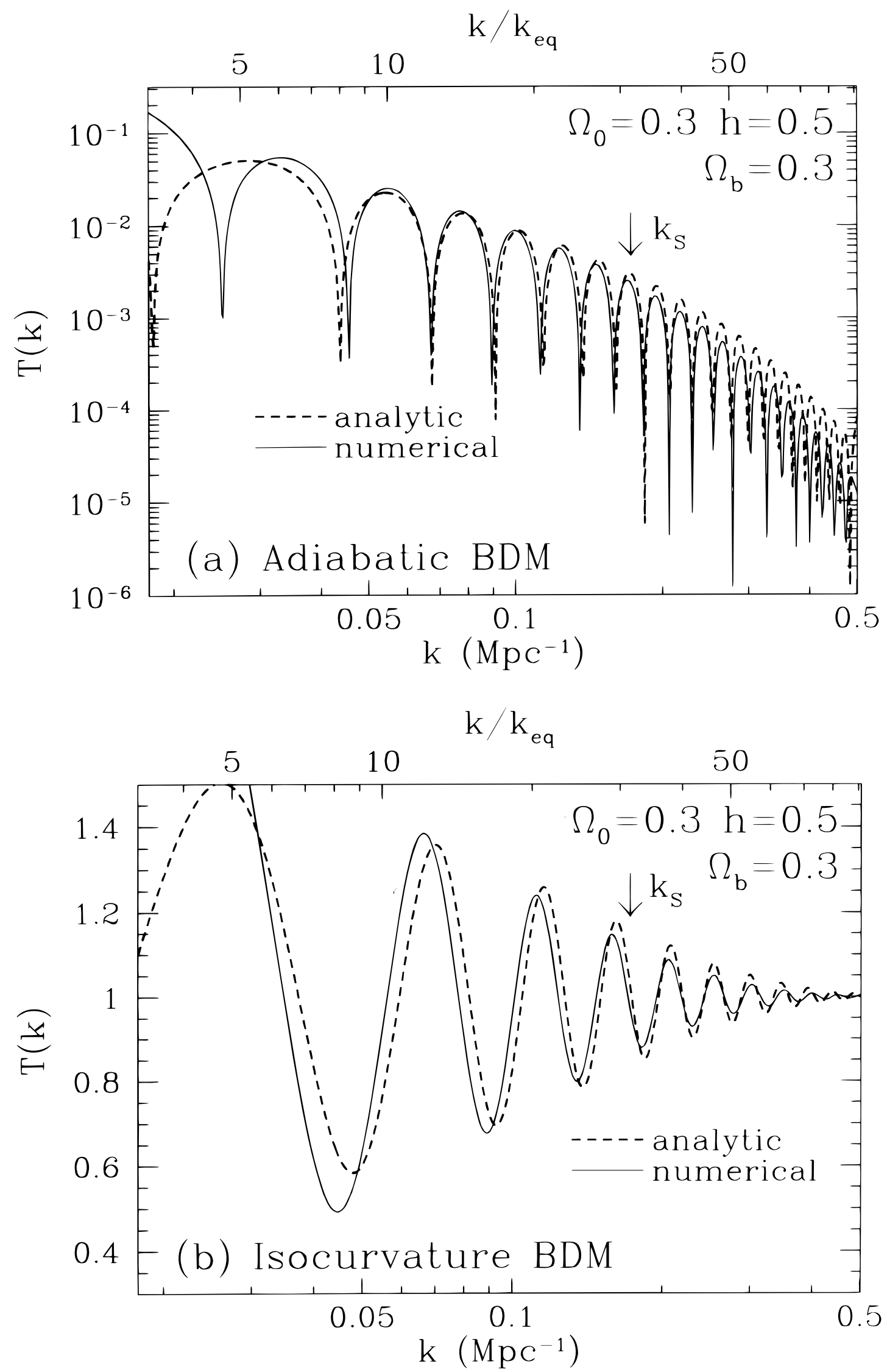

FIG. 5.-Matter transfer function. The analytic estimates of the intrinsic acoustic amplitude are good approximations for $k \gg k_{\text {eq. }}$. The Silk damping scale is approximated adequately although its value is underestimated by $\sim 10 \%$. For isocurvature BDM and adiabatic CDM, the acoustic contributions do not dominate the small-scale fluctuations. We have added in the contributions from the initial entropy fluctuations and the cold dark matter potentials according to the analytic treatment of Appendix E. 


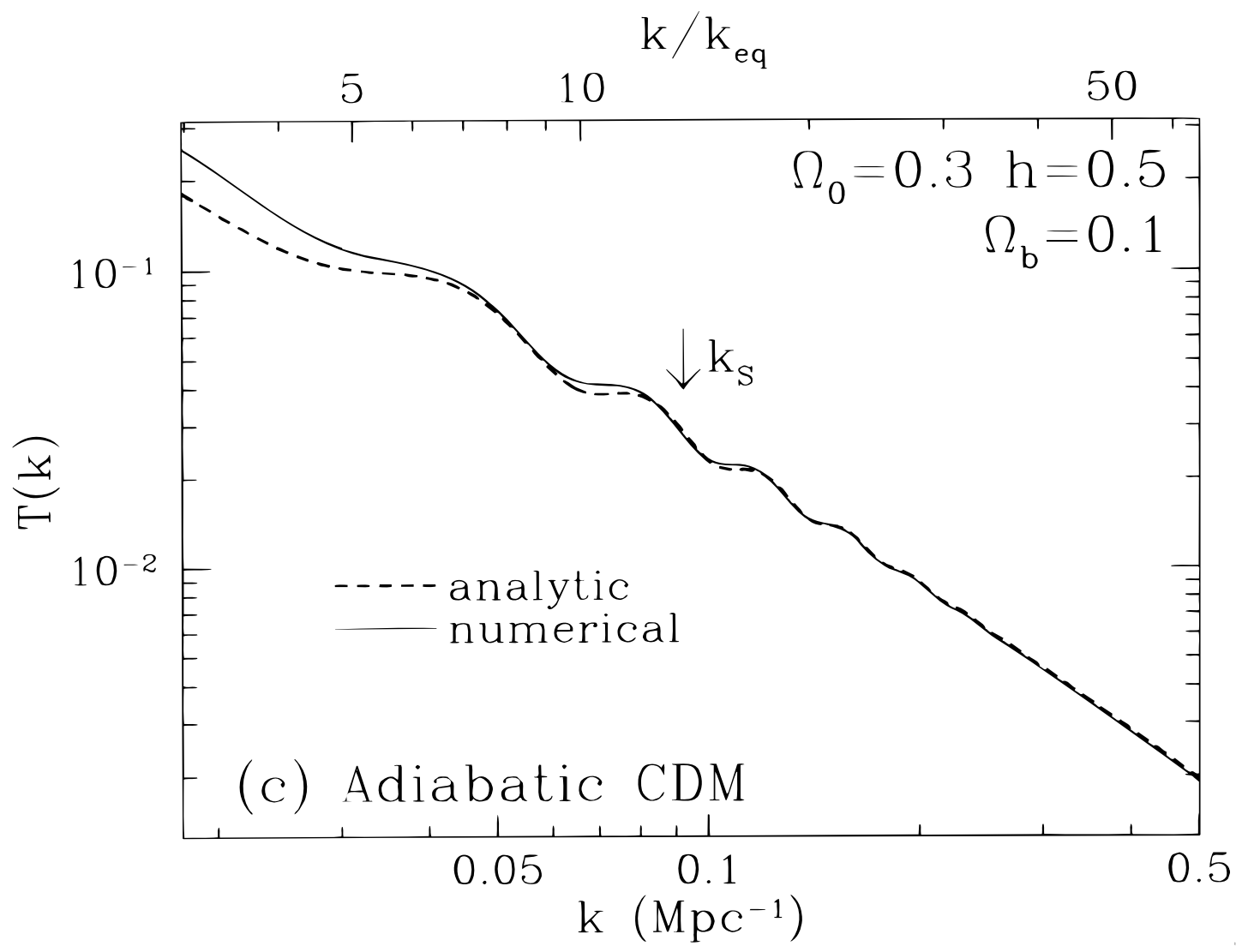

FIG. 5.-Continued

scaling relation is due to detailed matching of growing and decaying modes (see Appendix D, eq. [D18]). A highly accurate fitting formula for $\alpha$ in the general case is given in Appendix E, equation (E12). For the $\Omega_{b} \rightarrow 0$ limit, an exact expression in terms of elementary functions is given in Appendix D that improves the $10 \%$ accuracy of the standard BBKS fitting formula to better than the $1 \%$ level at small scales.

In Figure 6, we show the resulting evolution of a scale under the Silk damping length for which the baryon fluctuations at the drag epoch are negligible. To extend the scaling of equation (8) to larger scales for Figure 5, we have employed a generalization of the BBKS fitting function given in Appendix D. Notice that both (1) the change in the growth rate between equality and the drag epoch and (2) the fractional contribution of $\delta_{c}$ to $\delta_{m}$ at the drag epoch play a significant role in suppressing the final amplitude of matter fluctuations. Combined with the acoustic contributions from $\S 4$, this completes the matter transfer function in CDM models.

We can now address the question of when acoustic oscillations are prominent in the matter transfer function. The main effect is simply due to the density ratio $\rho_{b} / \rho_{c}=\Omega_{b} / \Omega_{c}$. However, the acoustic and cold dark matter contributions have a different dependence on scale. Relative to the cold dark matter, acoustic contributions scale as $\left(k \eta_{d}\right) \mathscr{D}_{b}$ due to the velocity overshoot and diffusion damping factors. Since $\mathscr{D}_{b}$ incorporates an exponential cutoff at the Silk scale $k_{\mathrm{S}}$ and velocity overshoot weights the spectrum toward small scales, acoustic contributions will be most visible just above the Silk scale. The relative contribution to the matter trans- fer function will therefore scale as $k_{\mathrm{S}} \eta_{d}$. Acoustic contributions increase in prominence if the Silk scale is small compared with the horizon at the drag epoch, i.e., in the high $\Omega_{b} h^{2}$ case. By including the suppression factor from equation (8) and numerical factors from Appendices B and $D$, the maximum ratio of the acoustic amplitude to the CDM contribution in the transfer function scales crudely as (see Appendix D, eq. [D31])

$$
0.4 k_{\mathrm{s}} \frac{\Omega_{b}}{\Omega_{c}}\left(\Omega_{0} h^{2}\right)^{-1}\left(1+24 \Omega_{0} h^{2}\right)^{-1 / 2}\left(1+32 \Omega_{b} h^{2}\right)^{-3 / 4} \alpha^{-1},
$$

where $k_{\mathrm{S}}$, here in units of $\mathrm{Mpc}^{-1}$, and $\alpha$ are given explicitly in Appendix E. This relation incorporates the following:

1. Baryon acoustic oscillations;

2. Baryon decoupling;

3. Silk damping;

4. Velocity overshoot;

5. Baryon gravitational instability;

6. Baryon infall into CDM wells whose depth depends on $(a)$ Acoustic feedback into the potential and (b) CDM self-gravity versus the expansion rate.

The relation combines these in a consistent manner.

\section{DISCUSSION}

We have established a framework for treating small-scale fluctuations in the realistic case of a coupled multifluid system. In the limit in which fluctuations crossed the horizon in the radiation-dominated epoch, closed-form 

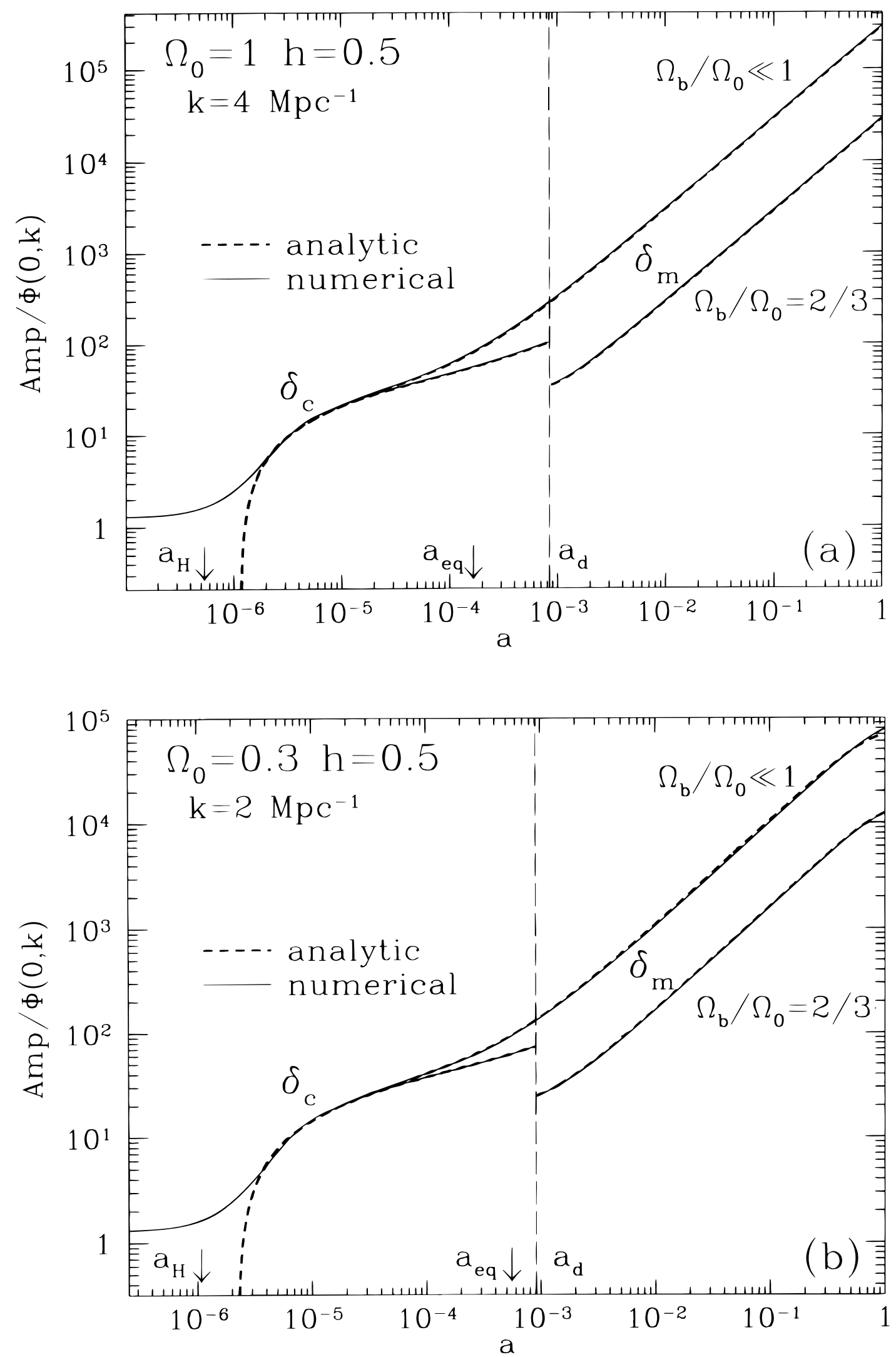

FIG. 6. CDM and matter fluctuation time evolution. The cold dark matter fluctuations are constant outside the horizon scale and experience a boost into a logarithmically growing mode at horizon crossing in the radiation-dominated epoch. Between equality and the drag epoch, the presence of baryons suppresses the growth rate of CDM fluctuations (see eq. [7]). By lowering $\Omega_{0} h^{2}$, this region of suppression can be reduced for fixed $\Omega_{b} / \Omega_{0}$. After the drag epoch, we plot $\delta_{m}=\left(\delta_{b} \rho_{b}+\delta_{c} \rho_{c}\right) /\left(\rho_{b}+\rho_{c}\right)$ rather than $\delta_{c}$ because the two components thereafter contribute similarly to the total growth. Baryons also lower the contribution of $\delta_{c}$ to $\delta_{m}$ at the drag epoch. 
analytic solutions are available. The fundamental elements uncovered by this approach are the boost at horizon crossing due to infall and dilation effects from potential decay, the source-free solution of the component evolution equations, the baryon drag on the photons, and the Compton drag on the baryons. Together they establish general consistency relations between the matter and the radiation power spectra as well as expose their sensitivity to changes in the background model.

Unlike the case of the matter fluctuations, decay in the potential results in an amplification of acoustic oscillations in the CMB. These opposing manifestations of the same physical effect provide both a measure and a powerful consistency check on the redshift of equality. Baryon drag displaces the zero point of the acoustic oscillations, which remains as a temperature shift even after the oscillations have damped by photon diffusion. Last scattering marks the end of the baryon drag epoch at which the acoustic oscillations with their characteristic diffusion damping scale are frozen into the photon spectrum. Correspondingly, at the end of the Compton drag epoch, the Silk-damped baryonic acoustic oscillations are frozen into the matter spectrum. We have provided convenient analytic fitting formulae for these quantities as a function of the matter and baryon content. These may be useful for the extraction of cosmological information from the CMB and matter power spectrum once observations become available.

The photon-baryon system also affects the growth of CDM fluctuations. First it stimulates growth through the horizon crossing boost. Subsequently, it affects the balance of the growth inhibiting expansion to the self-gravity of the CDM. We have obtained an analytic solution for adiabatic initial conditions and the exact general solution for the two effects, respectively. To simplify these expressions, we have also provided an accurate fitting form for the transfer function in terms of elementary functions. Growth suppression due to the presence of baryons has implications for the first generation of structure. The expressions derived here remain valid for linear perturbations to an arbitrarily small scale at which direct numerical calculations are impractical.

Baryonic acoustic oscillations are of course not prominent in presently popular models in which $\Omega_{b} / \Omega_{0} \ll 1$ due to the extra growth of the CDM fluctuations between horizon crossing and the drag epoch. However, they serve as a useful complement to their CMB counterpart if either $\Omega_{0} h^{2} \lesssim$ 0.05 or big bang nucleosynthesis constraints are too stringent (Gnedin \& Ostriker 1990). Indeed, there are tentative indications from cluster inventories that the baryon fraction may be as high as $15 \%$ (White et al. 1993). The presence or absence of acoustic oscillations in the observations of the CMB and large-scale structure will in the future provide a robust distinction between general classes of scenarios:

1. Oscillations in CMB and matter power spectra: standard recombination with $\Omega_{b} \gtrsim \Omega_{c}$;

2. Oscillations in CMB alone: standard recombination with $\Omega_{b} \ll \Omega_{c}$;
3. Oscillations in matter power spectra alone; early reionization with $\Omega_{b} \gtrsim \Omega_{c}$.

Large-scale structure observations already suggest there are no dramatic oscillations in the matter power spectrum as would be the case for classes (1) and (3) if $\Omega_{b} \gg \Omega_{c}$ (Peacock \& Dodds 1994). However, low-amplitude oscillations, as might be expected if $\Omega_{b} \sim \Omega_{c}$, remain possible. Of course, the exact form that these oscillations will take in the observations depends on issues such as redshift space distortions and nonlinear corrections.

If oscillations are discovered in neither spectra, the most natural conclusion is that our universe has $\Omega_{b} \ll \Omega_{c}$ and suffered early reionization. However, other possibilities include the formation of perturbations after recombination, reionization within an expansion time after the drag epoch, and equal or random stimulation of adiabatic and isocurvature mode acoustic fluctuations. ${ }^{3}$ These scenarios can be distinguished by measuring the location of the damping scale. All scenarios obeying standard recombination, regardless of the presence of actual peaklike structures, follow the scalings for the damping length discussed here. In all reionized models, the horizon at last scattering and at the drag epoch marks the damping scale for the photons and baryons, respectively.

If acoustic oscillations are discovered in both the CMB and large-scale structure power spectra, we will possess a strong consistency test for the dynamics of the expansion, i.e., a combination of the matter content, curvature, and cosmological constant, as well as the adiabatic or isocurvature nature of the initial fluctuations. Furthermore, the two contain complementary information on several specific fundamental cosmological parameters. The scale of the peaks in the matter power spectrum are determined mainly by the matter content $\Omega_{0} h^{2}$, whereas the angular scale of the CMB peaks is mostly sensitive to the curvature $1-\Omega_{0}$ $-\Omega_{\Lambda}$. The two damping lengths also probe different combinations of $\Omega_{0} h^{2}$ and $\Omega_{b} h^{2}$. The ratio of the peak heights to the underlying CDM contribution in the matter power spectrum probes $\Omega_{b} / \Omega_{0}$. Furthermore, by comparing similar scales, dependence on the initial power spectrum can be eliminated, providing a clean test of the whole gravitational instability paradigm.

We would like to acknowledge useful discussions with J. R. Bond, U. Seljak, J. Silk, and P. Steinhardt. W. H. would like to thank J. R. Bond for calling to his attention the enhancement of diffusion damping through polarization and $M$. White for pointing out several typos in the draft. W. H. was supported by grants from the NSF and the W. M. Keck Foundation.

\footnotetext{
${ }^{3}$ This may be accomplished by balancing the initial conditions or hypothesizing a gravitational forcing potential that is external to the linear photon-baryon-neutrino-CDM system, e.g., cosmological defects.
} 


\section{APPENDIX A}

\section{TIGHT COUPLING LIMIT}

\section{A1. EVOLUTION EQUATIONS}

The Fourier transform of the Newtonian temperature fluctuation can be broken up into Legendre moments, related to the direction cosines of the photon momenta $\gamma_{i}, \Theta(\eta, k, \gamma)=\Sigma_{l}(-i)^{l} \Theta_{l} P_{l}(k \cdot \gamma)$. The evolution equation for these moments is given by the Boltzmann hierarchy (Bond \& Efstathiou 1984; HSb):

$$
\begin{aligned}
& \dot{\Theta}_{0}=-\frac{k}{3} \Theta_{1}-\dot{\Phi}, \\
& \dot{\Theta}_{1}=k\left(\Theta_{0}+\Psi-\frac{2}{5} \Theta_{2}\right)-\dot{\tau}\left(\Theta_{1}-V_{b}\right), \\
& \dot{\Theta}_{2}=k\left(\frac{2}{3} \Theta_{1}-\frac{3}{7} \Theta_{3}\right)-\dot{\tau}\left(\frac{9}{10} \Theta_{2}-\frac{1}{10} \Theta_{2}^{Q}-\frac{1}{2} \Theta_{0}^{Q}\right), \\
& \dot{\Theta}_{l}=k\left(\frac{l}{2 l-1} \Theta_{l-1}-\frac{l+1}{2 l+3} \Theta_{l+1}\right)-\dot{\tau} \Theta_{l} \quad(l>2),
\end{aligned}
$$

if the ratio of the wavelength to the curvature scale is much smaller than the angle considered, i.e., $k \gg l(-K)^{1 / 2}$, where $K=-H_{0}^{2}\left(1-\Omega_{0}-\Omega_{\Lambda}\right)$. Recall that overdots are derivatives with respect to conformal time $\eta=\int d t / a$. Here $\Theta_{l}^{Q}$ is the $\mathrm{CMB}$ temperature perturbation in the Stokes parameter $Q$. It accounts for polarization generated by Compton scattering of anisotropic radiation. The metric fluctuations in the mode are given by $g_{00}=-(1+2 \Psi Y)$ and $g_{i j}=a^{2}(1+2 \Phi Y) \gamma_{i j}$, where $\gamma_{i j}$ is the three metric on a surface of constant curvature and $Y$ is a plane wave $e^{i \boldsymbol{k} \cdot \boldsymbol{x}}$ in flat space or more generally a $k$-eigenfunction of the Laplacian. The presence of the curvature perturbation $\Phi$ in the monopole equation represents the dilation effect. The form of the metric shows that it has the same origin as the photon redshift with the expansion. The gravitational potential $\Psi$ in the dipole or velocity equation accounts for gravitational infall or redshift.

The tight coupling approximation assumes that the Compton scattering rate $i$ is sufficiently rapid to equilibrate changes in the photon-baryon fluid. It is an expansion in the Compton scattering time $\tau^{-1}$, or more specifically the inverse of the optical depth through a wavelength $\dot{\tau} / k$ and through a period of the oscillation $\dot{\tau} / \omega=\dot{\tau} / k c_{s}>\dot{\tau} / k$, where

$$
c_{s}=\frac{1}{\sqrt{3(1+R)}},
$$

is the photon-baryon sound speed with $R=\frac{3}{4} \rho_{b} / \rho_{\gamma}$. To first order, only the $l=0$ monopole (with density fluctuation $\delta_{\gamma}=4 \Theta_{0}$ ) and $l=1$ dipole (with bulk velocity $V_{\gamma}=\Theta_{1}$ ) survive and one obtains the forced oscillator equation for acoustic waves in the photon-baryon fluid (HSa). ${ }^{4}$ To second order, the acoustic oscillations of the monopole and dipole are damped due to the imperfect coupling between the photons and baryons. Photon diffusion creates heat conduction through $\Theta_{1}-V_{b}$ and shear viscosity through $\Theta_{2}$ (Weinberg 1972; Bond 1996).

To close these equations, we need the continuity and Euler equations for the baryons,

$$
\begin{aligned}
& \dot{\delta}_{b}=-k V_{b}-3 \dot{\Phi}, \\
& \dot{V}_{b}=-\frac{\dot{a}}{a} V_{b}+k \Psi+\frac{\dot{\tau}\left(\Theta_{1}-V_{b}\right)}{R},
\end{aligned}
$$

the polarization hierarchy equations for the CMB (Bond \& Efstathiou 1984; Kosowsky 1996),

$$
\begin{aligned}
& \dot{\Theta}_{0}^{Q}=-\frac{k}{3} \Theta_{1}^{Q}-\dot{\tau}\left[\frac{1}{2} \Theta_{0}^{Q}-\frac{1}{10}\left(\Theta_{2}+\Theta_{2}^{Q}\right)\right], \\
& \dot{\Theta}_{1}^{Q}=k\left(\Theta_{0}^{Q}-\frac{2}{5} \Theta_{2}^{Q}\right)-\dot{\tau} \Theta_{1}, \\
& \dot{\Theta}_{2}^{Q}=k\left(\frac{2}{3} \Theta_{1}^{Q}-\frac{3}{7} \Theta_{3}^{Q}\right)-\dot{\tau}\left(\frac{9}{10} \Theta_{2}^{Q}-\frac{1}{10} \Theta_{2}-\frac{1}{2} \Theta_{0}^{Q}\right), \\
& \dot{\Theta}_{l}^{Q}=k\left(\frac{l}{2 l-1} \Theta_{l-1}^{Q}-\frac{l+1}{2 l+3} \Theta_{l+1}^{Q}\right)-\dot{\tau} \Theta_{l}^{Q} \quad(l>2),
\end{aligned}
$$

\footnotetext{
${ }^{4}$ In $\mathrm{HSa}$ and $\mathrm{HSb}$, we employed a hybrid gauge or "gauge-invariant" representation of density fluctuations for computational convenience. Since there are no benefits of this choice below the horizon, we work entirely in the Newtonian gauge in this paper. Only the definition of density fluctuations is affected: the total matter gauge $\Delta_{X}=\delta_{X}+3(\dot{a} / a)\left(1+p_{X} / \rho_{X}\right) V_{T} / k$, where $X$ represents any of the particle components.
} 


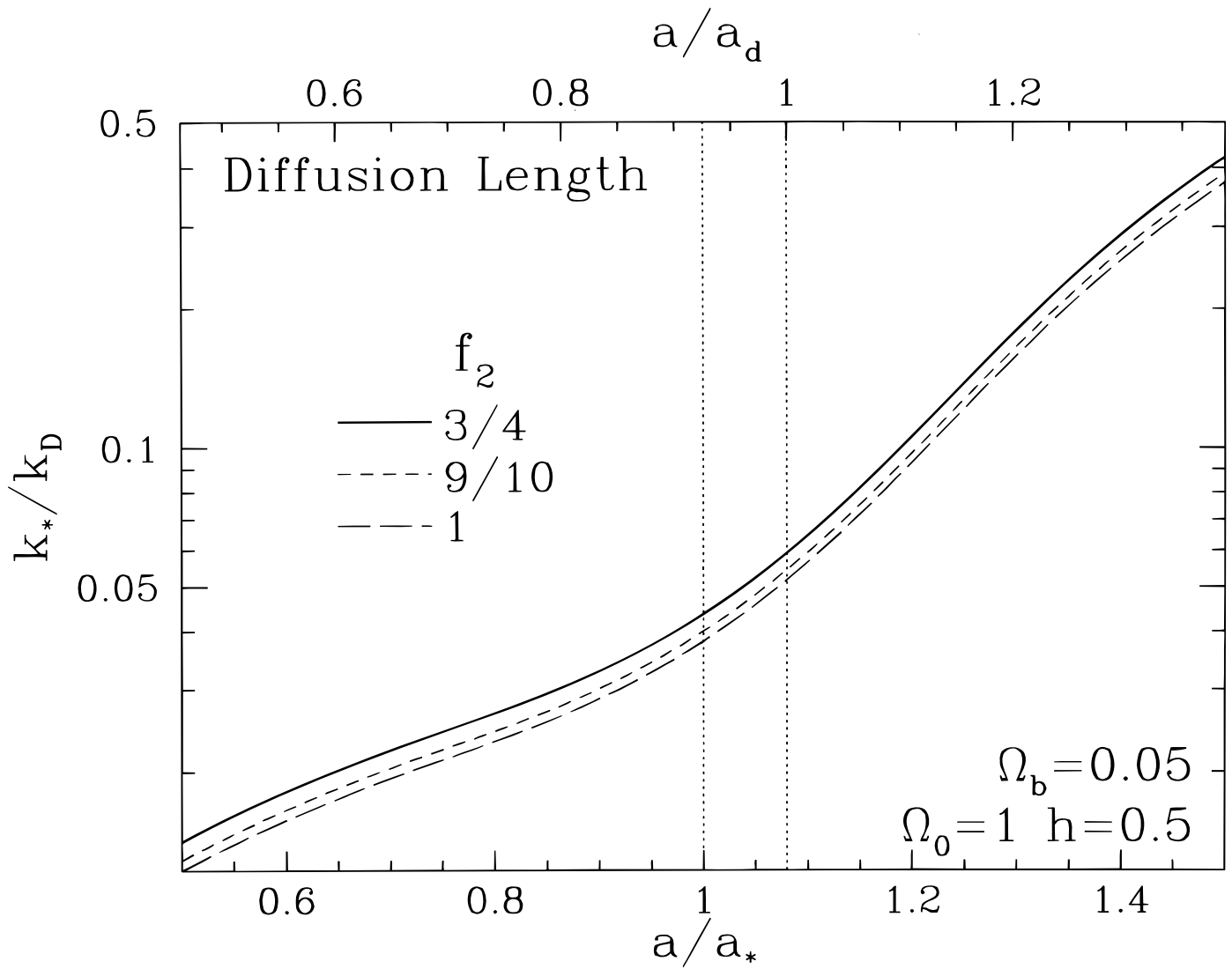

FIG. 7.-Photon diffusion scale. The photon diffusion scale grows rapidly near last scattering due to the increasing mean free path of the photons but remains well under the horizon scale $k_{*}^{-1}=\left.(\dot{a} / a)\right|_{a_{*}}$ at last scattering. The small difference between $a_{*}$ and $a_{d}$ is sufficient to cause a significant difference in the effective damping if $\Omega_{b} h^{2}$ differs substantially from the crossover point 0.03 . The inclusion of the angular dependence of Compton scattering enhances damping by a small factor $f_{2}=9 / 10$, as does the further inclusion of polarization $f_{2}=3 / 4$.

and the Einstein-Poisson equations for the metric or potential perturbations,

$$
k^{2} \Phi=4 \pi G a^{2} \rho_{T}\left[\delta_{T}+3 \frac{\dot{a}}{a}\left(1+w_{T}\right) \frac{V_{T}}{k}\right], \quad k^{2}(\Phi+\Psi)=-8 \pi G a^{2} p_{T} \Pi_{T},
$$

if $k \gg(-K)^{1 / 2}$. Here $w_{T}=p_{T} / \rho_{T}$, where the subscript $T$ denotes the total matter including all particle species and the total anisotropic stress is related to the radiation quadrupoles as

$$
p_{T} \Pi_{T}=\frac{12}{5}\left(p_{\gamma} \Theta_{2}+p_{\gamma} N_{2}\right),
$$

with $N_{2}$ as the neutrino temperature quadrupole. Notice that the baryon continuity equation can be rewritten as $\dot{\delta}_{b}=$ $-k\left(V_{b}-\Theta_{1}\right)+3 \dot{\Theta}_{0}$ because dilation effects on the photon temperature and baryon density fluctuations are analogous. This represents adiabatic evolution if $V_{b}=\Theta_{1}$.

\section{A2. ACOUSTIC DISPERSION RELATION}

Let us derive the dispersion relation for acoustic oscillations in the tight coupling limit. Consider first the effects of polarization. Since only the polarization monopole $\Theta_{0}^{Q}$ and quadrupole $\Theta_{2}^{Q}$ feed back into the temperature fluctuations, we may immediately expand the polarization hierachy in $\dot{\tau}^{-1}$ to obtain

$$
\Theta_{2}^{Q}=\Theta_{0}^{Q}=\frac{1}{4} \Theta_{2},
$$

which simplifies the temperature quadrupole evolution of equation (A1) to

$$
\dot{\Theta}_{2}=k\left(\frac{2}{3} \Theta_{1}-\frac{3}{7} \Theta_{3}\right)-\dot{\tau} f_{2} \Theta_{2},
$$

where $f_{2}=\frac{3}{4}$. Other approximations commonly used are $f_{2}=9 / 10$ for unpolarized radiation $($ Chibisov 1972$)$ and $f_{2}=1$ for further neglecting the angular dependence of Compton scattering (Weinberg 1972; Peebles 1980, p. 92; HSa). We keep the factor $f_{2}$ implicit so that the separation of effects can be read directly off the final results. Expanding the quadrupole equation (A8) to first order in $\dot{\tau}^{-1}$, we obtain

$$
\Theta_{2}=i^{-1} f_{2}^{-1} \frac{2}{3} k \Theta_{1}
$$

A second-order expansion for the quadrupole is not necessary because its effect on the fluid equations through $\dot{\Theta}_{1}$ is already of first order in $\dot{\tau}^{-1}$. 
On the other hand, a second-order expansion of the baryon Euler equation is necessary. Let us try a solution of the form $\Theta_{1} \propto \exp i \int \omega d \eta$ and ignore variations on the expansion timescale $\dot{a} / a$ in comparison with those at the oscillation frequency $\omega$. The electron velocity, obtained by iteration of the Euler equation, is to second order

$$
V_{b}=\Theta_{1}-\dot{\tau}^{-1} R\left(i \omega \Theta_{1}-k \Psi\right)-\dot{\tau}^{-2} R^{2} \omega^{2} \Theta_{1} .
$$

Substituting this into the photon dipole equation (A1) and eliminating the zeroth-order term yields

$$
i \omega(1+R) \Theta_{1}=k\left[\Theta_{0}+(1+R) \Psi\right]-\dot{\tau}^{-1} R^{2} \omega^{2} \Theta_{1}-\frac{4}{15} \dot{\tau}^{-1} f_{2}^{-1} k^{2} \Theta_{1} .
$$

This suggests that we try a solution of the form $\Theta_{0}+(1+R) \Psi \propto \exp i \int \omega d \eta$. Employing the monopole equation of(A1) and assuming again that variations at the oscillation frequency are sufficiently rapid that changes in $R, \Phi$, and $\Psi$ can be neglected, we obtain

$$
(1+R) \omega^{2}=\frac{k^{2}}{3}+i \dot{\tau}^{-1} \omega\left(R^{2} \omega^{2}+\frac{4}{15} k^{2} f_{2}^{-1}\right) .
$$

With the first-order relation $\omega^{2}=k^{2} / 3(1+R)$, the solution to the resultant quadratic equation is

$$
\omega= \pm \frac{k}{\sqrt{3(1+R)}}+i \frac{1}{6} k^{2} \dot{\tau}^{-1}\left[\frac{R^{2}}{(1+R)^{2}}+\frac{4}{5} f_{2}^{-1} \frac{1}{1+R}\right] .
$$

Thus, to second order acoustic oscillations are damped as exp $\left[-\left(k / k_{\mathrm{D}}\right)^{2}\right]$ with the damping length (Weinberg 1972; Kaiser 1983; Bond 1996)

$$
k_{D}^{-2}=\frac{1}{6} \int d \eta \frac{1}{i} \frac{R^{2}+4 f_{2}^{-1}(1+R) / 5}{(1+R)^{2}} .
$$

If $R \lesssim 1$, polarization and the angular dependence of Compton scattering enhances damping through the generation of viscosity (see Fig. 7). Viscosity is related to photon diffusion because the quadrupole and higher moments are generated as photons from regions of different temperatures meet.

\section{A3. BARYON DRAG AND THE ADIABATIC INVARIANT}

The temperature perturbation oscillates around $\Theta_{0}+(1+R) \Psi=0$ due to the baryon drag effect. This can be understood more easily by examining the first-order equation from which it originates,

$$
(1+R) \ddot{\Theta}_{0}+\frac{k^{2}}{3} \Theta_{0} \simeq-\frac{k^{2}}{3}(1+R) \Psi,
$$

ignoring slow changes in $R, \Phi$, and $\Psi$ from the expansion. Notice that $m_{\text {eff }}=(1+R)$ plays the role of the effective mass of the oscillator and the gravitational potential provides the effective acceleration through infall. The photon pressure acts as the restoring force and is independent of the baryon content $R$.

Equation (A15) has a simple solution

$$
\Theta_{0}=\left[\Theta_{0}(0, k)+(1+R) \Psi\right] \cos (\omega \eta)+\frac{1}{\omega} \dot{\Theta}_{0}(0, k) \sin (\omega \eta)-(1+R) \Psi,
$$

where the frequency $\omega=k /\left(3 m_{\mathrm{eff}}\right)^{1 / 2}=k /[3(1+R)]^{1 / 2}$ in agreement with the first-order dispersion relation of equation (A13). This solution describes an oscillator whose zero point has been displaced by $-m_{\mathrm{eff}} \Psi=-(1+R) \Psi$ due to the gravitational force (see Fig. 8). The photons, although massless, suffer infall effects due to gravitational blueshift. Since this is exactly cancelled as the photons stream out of the potential wells, we can consider $\Theta_{0}+\Psi$ as the effective temperature perturbation. Thus, this part of the zero point shift has no net effect. However, the baryons also contribute to the effective mass of the fluid. Since the photons and baryons are tightly coupled, baryonic infall drags the photons into potential wells and contributes $-R \Psi$ to the displacement. Notice that baryon drag also increases the amplitude of the cosine oscillation because the initial conditions $\Theta(0, k)$ represent a greater displacement from the zero point. Thus, baryon drag accounts for both the alternating peak heights of the acoustic oscillations and their enhancement with $\Omega_{b} h^{2}(\mathrm{HSa})$. After diffusion damping has eliminated the oscillations themselves, the zero-point shift $-R \Psi$ remains. Of course, for adiabatic BDM models $\Psi$ is also reduced to zero by the diffusion.

In reality, the variation of oscillator parameters, such as the effective mass and gravitational force, on the expansion timescale cannot be ignored over many periods of the oscillation. From equations (A1) and (A3), the full first-order equation is

$$
\frac{d}{d \eta}(1+R) \dot{\Theta}_{0}+\frac{k^{2}}{3} \Theta_{0}=-\frac{k^{2}}{3}(1+R) \Psi-\frac{d}{d \eta}(1+R) \dot{\Phi}
$$

where the addition of the space curvature term comes from the dilation effect $\dot{\Theta}_{0}=-\dot{\Phi}$. Notice that the left-hand side is precisely the equation of an oscillator with a time-varying mass $m_{\text {eff }}=(1+R)$. The homogeneous equation can be solved by employing the fact that variations over a single period of the oscillation are small. The adiabatic invariant associated with an oscillator is given by the energy $E=\frac{1}{2} m_{\mathrm{eff}} \omega^{2} A^{2}$ over the frequency $\omega$. Therefore, the amplitude scales as 

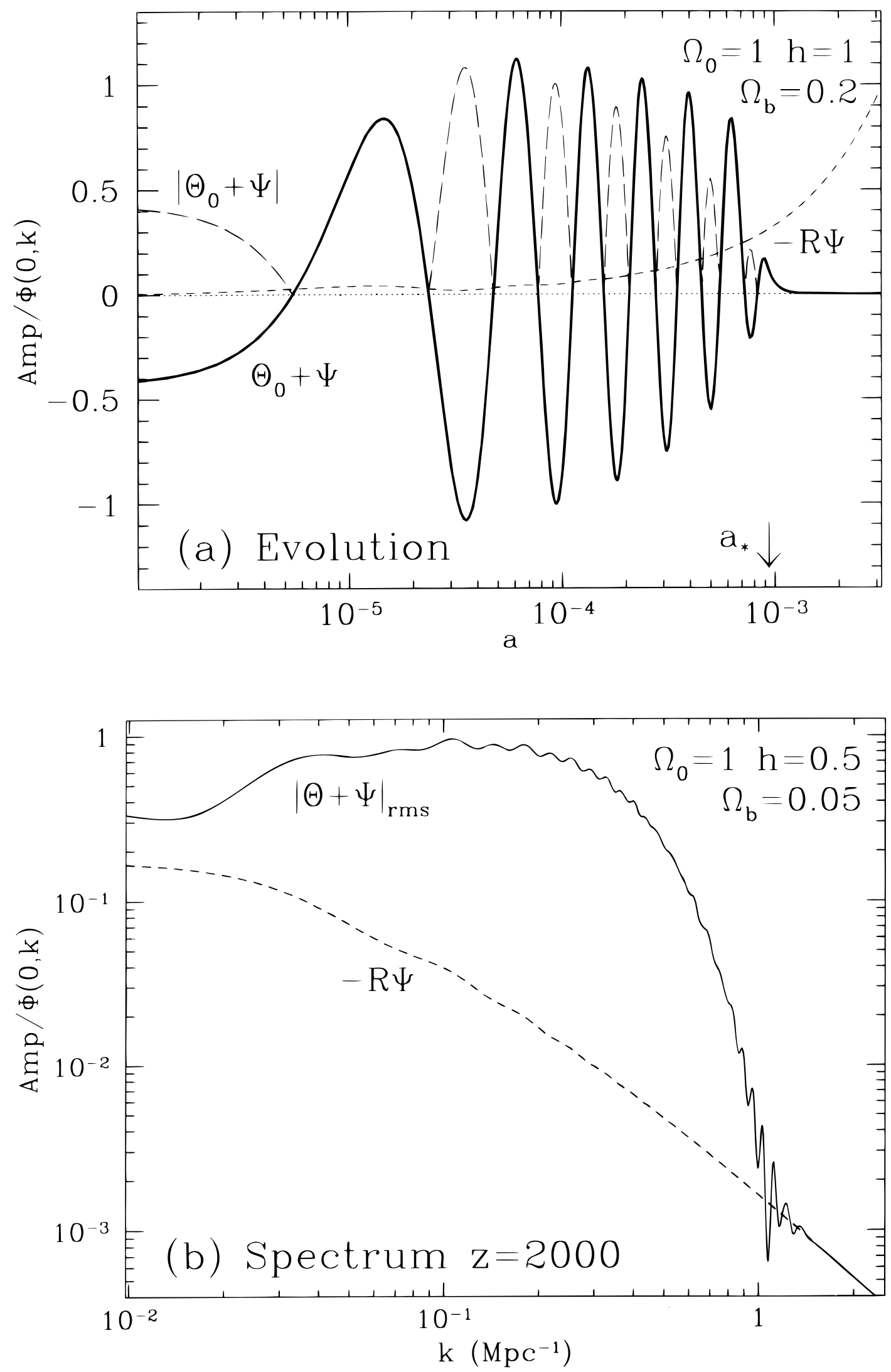

FIG. 8.-Baryon drag effect in adiabatic CDM models. (a) Baryons cause a drag effect on the photons, leading to a temperature enhancement of $-R \Psi=|R \Psi|$ inside potential wells, which shifts the zero point of the oscillation (short-dashed lines). (b) This contribution yields alternating peak heights in the rms and is also retained after diffusion damping. Here numerical results are displayed. 
$A \propto \omega^{1 / 2} \propto(1+R)^{-1 / 4}$. This yields fundamental solutions of the form $(1+R)^{-1 / 4} \exp \left( \pm i \int \omega d \eta\right)($ Peebles $\&$ Yu 1970). The phase integral can be performed analytically,

$$
\int \omega d \eta=k \int c_{s} d \eta=k r_{s}
$$

where $r_{s}$ is the sound horizon given in equation (1).

\section{A4. SUMMARY}

Several points are worth emphasizing:

1. The first-order dispersion relation for acoustic oscillations is $\omega=k c_{s}=k /[3(1+R)]^{1 / 2}$.

2. Slow changes in the baryonic contribution to the effective mass cause the temperature oscillation to decay as $(1+R)^{-1 / 4}$.

3. The oscillation phase is related to the sound horizon $r_{s}=\int c_{s} d \eta$ by $\int \omega d \eta=k r_{s}$.

4. Photon diffusion alters the dispersion relation and leads to exponential damping.

5. The damping length increases roughly as $\lambda_{D} \sim k_{D}^{-1} \sim(\eta / \tau)^{1 / 2}$ or the geometric mean of the conformal time and the Compton mean free path, as one expects of a random walk.

6. If it is well under the horizon, the damping length surpasses the wavelength when $\dot{\tau} / k=k \eta \gg 1$ or when the optical depth through a wavelength is still high.

7. The angular dependence of Compton scattering and polarization increases the damping length when $R \lesssim 1$.

8. The zero point of oscillations in $\Theta_{0}+\Psi$ is $-R \Phi$ due to baryon drag and remains as a temperature shift after diffusion damping.

\section{APPENDIX B}

\section{HORIZON CROSSING}

\section{B1. PHOTON-BARYON SYSTEM}

The amplitude of the acoustic oscillation is determined by the growth of fluctuations before and in particular during the epoch when the scale crosses the horizon. Since the second-order tight coupling expansion yields just a multiplicative diffusion damping factor, let us obtain the first-order solution, which we denote by carets. The full solution can be constructed through the relations

$$
\begin{aligned}
\Theta_{0}+\Psi & =\left[\hat{\Theta}_{0}+(1+R) \Psi\right] \exp \left[-\left(k / k_{D}\right)^{2}\right]-R \Psi, \\
\Theta_{1} & =\hat{\Theta}_{1} \exp \left[-\left(k / k_{D}\right)^{2}\right], \\
\delta_{b} & =3 \Theta_{0}+S(0, k), \\
k V_{b} & =k \Theta_{1}=-\dot{\Theta}_{0}-\dot{\Phi},
\end{aligned}
$$

where recall $S(0, k)$ is the initial entropy perturbation in the photon-baryon system $S(0, k)=\delta_{b}(0, k)-\frac{3}{4} \delta_{\gamma}(0, k)$. Near or above the horizon at $\eta_{*}$, where the sources from potential growth and decay drive the oscillator, the full formalism of HSa is needed to follow the fluctuations accurately. However, we are interested only in small-scale fluctuations that enter the horizon well before last scattering. Since radiation pressure prevents the growth of fluctuations in the radiation-dominated epoch, the gravitational potentials decay away after horizon crossing. On small scales, the acoustic oscillations therefore experience a boost at horizon crossing and thereafter settle into pure modes $\Theta_{0}(\eta, k)=(1+R)^{-1 / 4}\left[C_{A}(k) \cos \left(k r_{s}\right)+C_{I}(k) \sin \left(k r_{s}\right)\right]$. Equation (A17) yields exact solutions for $C_{A}$ and $C_{I}$ for the fundamental adiabatic and isocurvature modes of the fluctuation if anisotropic stress $\Pi_{T}$ is ignored so that $\Psi=-\Phi$. The adiabatic modes arises from an initial curvature perturbation $\Phi(0, k)$, whereas the isocurvature mode arises from an initial entropy perturbation $S(0, k)$. The solutions are (Kodama \& Sasaki 1986; $\mathrm{HSb})$

$$
\lim _{\Pi \rightarrow 0} C_{A}(k)=\frac{3}{2} \Phi(0, k) \quad \text { adiabatic, } \quad \lim _{\Pi \rightarrow 0} C_{I}(k)=-\frac{\sqrt{6}}{4} \frac{k_{\text {eq }}}{k} S(0, k) \text { isocurvature, }
$$

where $k_{\mathrm{eq}}=\left(2 \Omega_{0} H_{0}^{2} / a_{\mathrm{eq}}\right)^{1 / 2}=9.67 \times 10^{-2} \Omega_{0} h^{2}\left(1-f_{v}\right)^{1 / 2} \Theta_{2.7}^{-2} \mathrm{Mpc}^{-1}$ is the wavenumber that passes the horizon at equality. Recall that $f_{v}=\rho_{v} /\left(\rho_{\gamma}+\rho_{v}\right)$. The two modes stimulate pure cosine and sine modes because the gravitational forcing function yields near-resonant driving with the phase fixed by $\Phi(0, k)=$ constant and $\Phi(0, k)=0$, respectively (HSb; Hu \& White 1996). The amplitude of the fluctuations is easy to understand qualitatively. For the adiabatic case, $\Theta_{0}(0, k)=\frac{1}{2} \Phi(0, k)$. If photon streaming is ignored, $\dot{\Theta}=-\dot{\Phi}$, the dilation effect would raise the amplitude to $\Theta_{0}(\eta, k)=\frac{1}{2} \Phi(0, k)-\Phi(\eta, k)+\Phi(0, k)=$ $\frac{3}{2} \Phi(0, k)$. Note that a decaying potential boosts the acoustic amplitude due to the gravitational forcing effect. A similar analysis for the isocurvature mode accounting for potential growth outside the horizon explains the isocurvature amplitude (HSb).

The effect of anisotropic stress can be considered as a perturbation ( $\mathrm{HSa}$ ). The dominant term comes from the neutrino quadrupole because photon anisotropies are damped exponentially with optical depth before last scattering. The order or 
magnitude can be simply read off the initial conditions for the growing mode of the perturbation,

$$
\Theta_{0}(0, k)=-\frac{1}{2} \Psi(0, k)=\frac{1}{2}\left(1+\frac{2}{5} f_{v}\right)^{-1} \Phi(0, k) \quad \text { adiabatic },
$$

or $\Theta_{0}(0, k)=\Psi(0, k)=\Phi(0, k)=0$ and

$$
\begin{aligned}
& \dot{\Theta}_{0}(0, k)=\frac{\sqrt{2}}{16} k_{\mathrm{eq}}\left(1+\frac{2}{15} f_{v}\right) S(0, k), \\
& \dot{\Phi}(0, k)=\frac{\sqrt{2}}{16} k_{\mathrm{eq}}\left(1+\frac{2}{15} f_{v}\right) S(0, k) \text { isocurvature } \\
& \dot{\Psi}(0, k)=-\frac{\sqrt{2}}{16} k_{\mathrm{eq}}\left(1-\frac{6}{15} f_{v}\right) S(0, k) .
\end{aligned}
$$

Indeed, we find that the adiabatic amplitude is well approximated by

$$
C_{A}(k)=\frac{3}{2}\left(1+\frac{2}{5} f_{v}\right)^{-1} \Phi(0, k) \text { adiabatic , }
$$

and likewise

$$
C_{I}(k)=-\frac{\sqrt{6}}{4} \frac{k_{\mathrm{eq}}}{k}\left(1-\frac{4}{15} f_{v}\right) S(0, k) \text { isocurvature, }
$$

for the isocurvature mode. Explicitly, the first-order acoustic solution is

$$
\begin{aligned}
\hat{\Theta}_{0}+\Psi & \simeq \hat{\Theta}_{0}=(1+R)^{-1 / 4}\left[C_{A} \cos \left(k r_{s}\right)+C_{I} \sin \left(k r_{s}\right)\right], \\
\hat{\Theta}_{1} & =\hat{V}_{b}=-\sqrt{3}(1+R)^{-3 / 4}\left[C_{A} \sin \left(k r_{s}\right)+C_{I} \cos \left(k r_{s}\right)\right] .
\end{aligned}
$$

In Figure 1, we display an adiabatic example. In this case, it is also useful to compare the acoustic amplitude to the Sachs-Wolfe effect (Sachs \& Wolfe 1967) $|\Theta+\Psi|_{\mathrm{rms}}\left(\eta_{0}, k\right)=\frac{1}{3} \Psi\left(\eta_{0}, k\right)$. With the relation (see HSa, eq. [A18]),

$$
\Psi\left(\eta_{0}, k\right)=-\frac{9}{10}\left(1+\frac{4}{15} f_{v}\right)^{-1}\left(1+\frac{2}{5} f_{v}\right)^{-1} \Phi(0, k),
$$

valid at large scales $k \ll k_{\text {eq }}$, the relative amplitude becomes

$$
\left|3 C_{A}(k) / \Psi\left(\eta_{0}, k\right)\right|=5\left(1+\frac{4}{15} f_{v}\right)^{-1}
$$

and it represents a significant boost.

\section{B2. CDM COMPONENT}

The evolution of the cold dark matter fluctuations in the presence of acoustic oscillations is also interesting and relevant for determining the small-scale behavior of the matter transfer function (see Appendix D). The cold dark matter evolution equations are of the same form as the baryon continuity and Euler equations save for the absence of coupling to the photons,

$$
\ddot{\delta}_{c}+\frac{\dot{a}}{a} \dot{\delta}_{c}=-k^{2} \Psi-3 \ddot{\Phi}
$$

In the radiation-dominated epoch, the metric terms on the right-handside are dominated by perturbations in the radiation and may be considered as external driving forces. The homogeneous equation has two fundamental solutions $\delta_{c} \propto\{\ln a, 1\}$. The particular solution is constructed via Green's method,

$$
\delta_{c}=C_{1} \ln a+C_{2}+\int_{0}^{\eta}\left[\ln a^{\prime}-\ln a\right] \frac{a^{\prime}}{\dot{a}^{\prime}}\left(k^{2} \Psi+3 \ddot{\Phi}\right) d \eta^{\prime} .
$$

Adiabatic initial conditions require $C_{1}=0$ and $C_{2}=3 \Theta_{0}(0, k)$. Thus, $\delta_{c}$ remains constant outside the horizon and then gets a kick from infall and dilation that generates a logarithmic growing mode. Since the behavior of the potentials is self-similar in $k$, i.e., they are constant outside the horizon and decay to zero as $a^{-2}$ inside of it, their effect on $\delta_{c}$ is the same for all $k$. Once the potentials have decayed to zero, $\delta_{c}$ settles into the logarithmic growing mode as

$$
\delta_{c} \simeq I_{1} \Phi(0, k) \ln \left(I_{2} \frac{a}{a_{H}}\right) \quad a_{H} \ll a \ll a_{\mathrm{eq}},
$$

where horizon crossing occurs at

$$
\begin{aligned}
\frac{a_{H}}{a_{\mathrm{eq}}} & =\frac{1+\sqrt{1+8\left(k / k_{\mathrm{eq}}\right)^{2}}}{4\left(k / k_{\mathrm{eq}}\right)^{2}}, \\
& \simeq \frac{\sqrt{2}}{2} \frac{k_{\mathrm{eq}}}{k} \quad k \gg k_{\mathrm{eq}} .
\end{aligned}
$$


By numerical calculation of the integrals in equation (B11), we obtain

$$
\begin{aligned}
& I_{1}=9.11\left(1+0.128 f_{v}+0.029 f_{v}^{2}\right), \\
& I_{2}=0.594\left(1-0.631 f_{v}+0.284 f_{v}^{2}\right),
\end{aligned}
$$

valid at the $1 \%$ or better level for the full range $0 \leq f \leq 1$. As we shall see in Appendix $\mathrm{D}$, this solution can be joined onto the growing mode in the matter-dominated epoch to describe the full time evolution of the CDM fluctuations.

\section{APPENDIX C}

\section{DECOUPLING}

\section{C1. PHOTON DECOUPLING}

The tight coupling approximation is strictly valid only well before decoupling. However, the acoustic modes may be simply joined onto the free-streaming solutions once diffusion damping near decoupling has been taken into account. The full Boltzmann hierarchy has the formal solution (see HSa, eq. [11])

$$
(\Theta+\Psi)\left(\eta_{0}, k, \mu\right)=\int_{0}^{\eta_{0}} d \eta\left[\left(\Theta_{0}+\Psi-i \mu V_{b}\right) \dot{\tau}-\dot{\Phi}+\dot{\Psi}\right] e^{-\tau} e^{i k \mu\left(\eta-\eta_{0}\right)},
$$

where $k \mu=\boldsymbol{k} \cdot \gamma$ and curvature has been neglected. The terms in parentheses contribute at last scattering due to weighting by the visibility function $\mathscr{V}_{\gamma}=\dot{\tau} e^{-\tau}$, and the integrated Sachs-Wolfe metric terms play a role between last scattering and the present. This formal solution is made practical by replacing the sources $\Theta_{0}$ and $V_{b}$ with their acoustic solution at last scattering.

It may seem that employing the tight coupling solution through decoupling would lead to erroneous results. In particular, the damping approximation should break down if the optical depth through a wavelength drops below unity. However, let us examine the tight coupling solution more carefully. Equation (B1) implies

$$
[\Theta+\Psi]\left(\eta_{0}, k, \mu\right)=\int_{0}^{\eta_{0}} d \eta\left(\hat{\Theta}_{0}+\Psi-i \mu \hat{V}_{b}\right)\left\{\mathscr{V}_{\gamma} e^{-\left[k / k_{D}(\eta)\right]^{2}}\right\} e^{i k \mu\left(\eta-\eta_{0}\right)}+\int_{0}^{\eta_{0}} d \eta R \Psi\left\{e^{-\left[k / k_{D}(\eta)\right]^{2}}-1\right\} \mathscr{V}_{\gamma} e^{i k \mu\left(\eta-\eta_{0}\right)} .
$$

Although baryon drag effects such as acoustic displacement and enhancement are already encorporated in the acoustic solution $\widehat{\Theta}_{0}$ (see eq. [A16]), the residual $R \Psi$ term appears beneath the diffusion scale. Let us ignore this for the moment. The effective visibility for the acoustic oscillations is given by

$$
\hat{\mathscr{V}}_{\gamma}=\mathscr{V}_{\gamma} e^{-\left[k / k_{D}(\eta)\right]^{2}} .
$$

This function is plotted for a given model in Figure 2. Unlike $\mathscr{V}_{\gamma}, \hat{\mathscr{V}}_{\gamma}$ is damped exponentially at late times by the growing diffusion length and thus peaks at earlier times. Note that a $10 \%$ shift in redshift represents a factor of 3 in optical depth near last scattering. Thus, the region in which we expect the approximation to break down is given little weight in the integral. More specifically, the exponential damping ensures that most contributions come from before the epoch at which the diffusion length surpasses the wavelength. As we have seen in Appendix A, the optical depth through a wavelength is high at this time and justifies the tight coupling expansion.

The damping of acoustic modes through last scattering can occur in general due to two different mechanisms working in equation (C2): diffusion and cancellation. However, the effects are of greatly unequal magnitude. Cancellation occurs because on small scales many wavelengths of the perturbation span the Compton visibility function. Photons that scattered last at the crests of the perturbations will have their effect canceled by those that scattered at the troughs. Mathematically this occurs in equation $(\mathrm{C} 2)$ because the oscillating plane wave is integrated over the visibility function. Cancellation leads to a power-law damping of fluctuations as the scale decreases below the width of the visibility function. However, in the case of diffusion damped acoustic contributions, it is not the width of the Compton visibility function $\mathscr{V}_{\gamma}$ that is relevant but rather the acoustic visibility function $\hat{\mathscr{V}}_{\gamma}$. As one goes to smaller and smaller scales (high $k$ ), the width of this function decreases as well. Thus, even at high $k$ the cancellation regime is never fully reached, and one may approximate the integral (C2) by replacing $\hat{\mathscr{V}}_{\gamma}$ by a delta function, i.e.,

$$
(\Theta+\Psi)\left(\eta_{0}, k, \mu\right) \simeq\left(\hat{\Theta}_{0}+\Psi-i \mu \hat{V}_{b}\right)\left(\eta_{*}, k\right) e^{i k \mu\left(\eta_{*}-\eta_{0}\right)} \mathscr{D}_{\gamma}(k),
$$

where

$$
\mathscr{D}_{\gamma}(k)=\int_{0}^{\eta_{0}} d \eta \hat{\mathscr{V}}_{\gamma}=\int_{0}^{\eta_{0}} d \eta \mathscr{V}_{\gamma} e^{-\left[k / k_{D}(\eta)\right]^{2}} .
$$

The observable anisotropy follows by decomposing equation (C4) into Legendre moments $\Theta=\Sigma(-i)^{l} \Theta_{l} P_{l}(\mu)$ and summing over $k$-modes $C_{l}=(2 / \pi) \int k^{3}\left|\Theta_{l}\left(\eta_{0}, k\right) /(2 l+1)\right|^{2} d \ln k$ (HSa). Decoupling thus increases the effective diffusion damping length due to the corresponding increase in the mean free path of the photons. The result is a near-exponential damping with scale that overwhelms completely the small residual cancellation damping. 

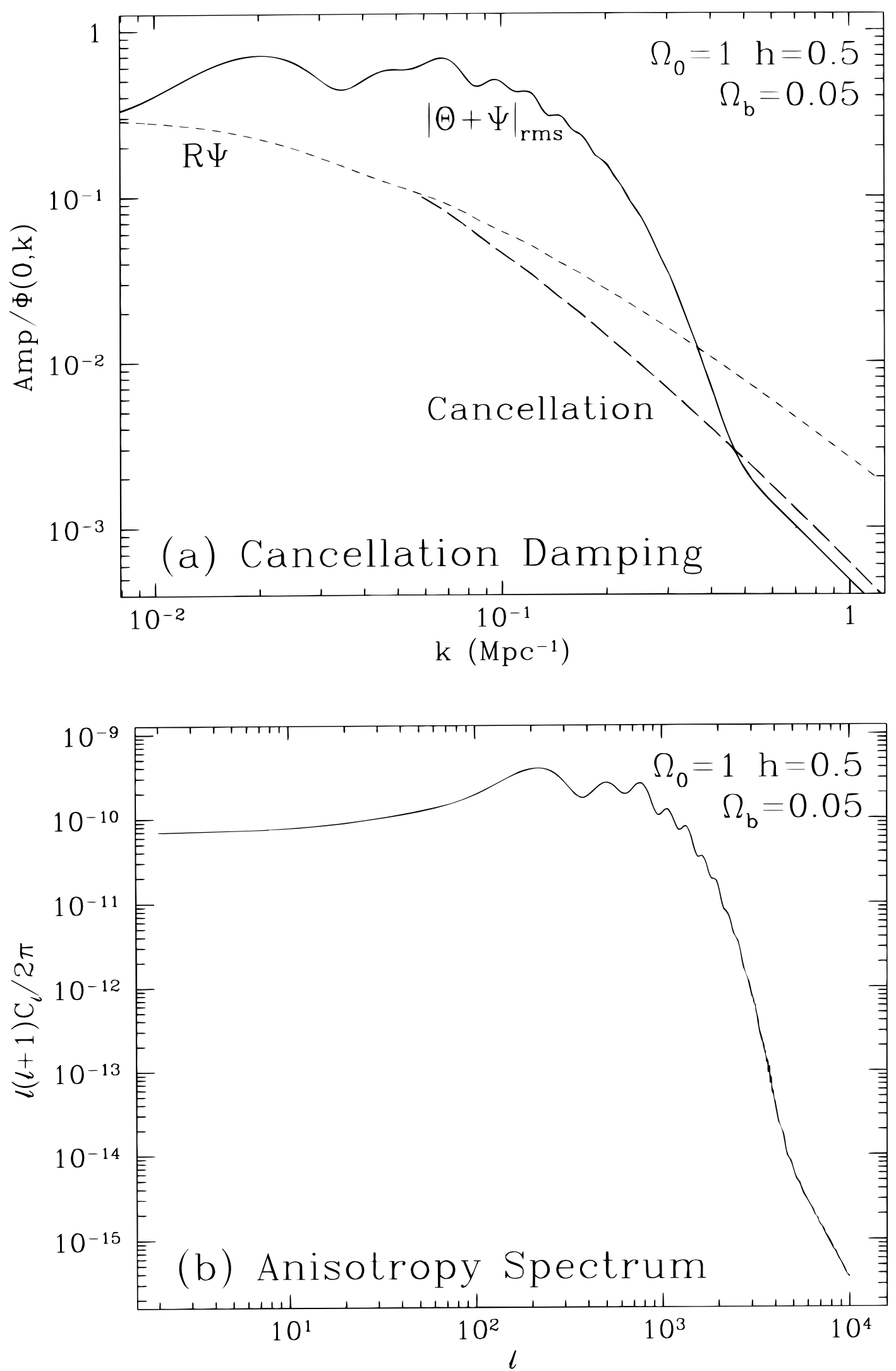

FIG. 9.- Residual baryon drag effect after last scattering in an adiabatic CDM model. On scales under the width of the visibility function, cancellation between contributions that came from potential wells and hills at last scattering damps fluctuations from the baryon drag effect. Note that cancellation damping is weak and scales as $\left(k \eta_{*}\right)^{-1 / 2}$, in contrast to the exponential diffusion damping. Projection relates the rms fluctuation in (a) to the anisotropy power spectrum in $(b)$. 
Cancellation damping does occur for the baryon drag effect $-R \Psi$ in equation $(\mathrm{C} 2)$ that remains after diffusion damping. The amplitude of the resultant fluctuations can be estimated by noting that

$$
(\Theta+\Psi)\left(\eta_{0}, k, \mu\right)=-\int_{0}^{\eta_{0}} d \eta \mathscr{V}_{\gamma} R \Psi e^{i k \mu\left(\eta-\eta_{0}\right)},
$$

is approximately a Fourier transform. Employing Parceval's theorem, we obtain

$$
\left|\Theta_{0}+\Psi\right|_{\mathrm{rms}}^{2} \equiv \frac{1}{2} \int_{-1}^{1}|\Theta+\Psi|^{2} d \mu \simeq \frac{\pi}{k} \int_{0}^{\eta_{0}}\left|R \Psi \mathscr{V}_{\gamma}\right|^{2} d \eta \simeq \frac{\pi}{k}\left|\Psi\left(\eta_{*}, k\right)\right|^{2} \int_{0}^{\eta_{0}}\left(R \mathscr{V}_{\gamma}\right)^{2} d \eta
$$

Thus, the contribution to the rms is suppressed by roughly $\left(k \eta_{*}\right)^{-1 / 2}$ due to cancellation. Note that the residual baryon drag effect only appears in models in which $\Psi$ itself is not damped away by diffusion, e.g., adiabatic CDM and isocurvature BDM models. In Figure $9 a$, we display this effect. The amplitude of the effect is overestimated slightly because baryon drag weakens through last scattering. Its effect on the anisotropy is shown in Figure $9 b$ and can be obtained analytically in a computationally simple matter through the approximations of $\mathrm{Hu} \&$ White (1996). Since it is unlikely to be observable due to effects in the foreground of last scattering, we omit a detailed calculation here.

\section{C2. BARYON DECOUPLING}

The formal solution to the baryon Euler equation (A3) is

$$
a V_{b}=\int_{0}^{\eta} d \eta^{\prime} a\left(\dot{\tau}_{d} \Theta_{1}+k \Psi\right) e^{-\tau_{d}},
$$

where $\dot{\tau}_{d}=\dot{\tau} / R$ and quantities in the integrand are evaluated at $\eta^{\prime}$. The two source terms are the Compton drag effect and infall into potential wells. The former forces the baryon velocity to follow the photon dipole (velocity) at high drag optical depth $\tau_{d}$. The presence of the scale factor $a$ in the equation represents the fact that baryon velocities decay as $a^{-1}$ in the absence of sources. Since $\dot{\tau}_{d} e^{-\tau_{d}}$ is very nearly a delta function with respect to variations on the expansion timescale, this equation is conceptually identical to its photon analog $(\mathrm{C} 1)$ with the replacement $\dot{\tau} \rightarrow \dot{\tau}_{d}$. The plane-wave factor is absent for the baryons because their particle velocities are low and the streaming can be neglected in comparison to the wavelength. Drag depth unity $\tau_{d}\left(z_{d}\right)=1$ marks the transition between the drag and infall epochs. For $\Omega_{b} h^{2} \gtrsim 0.03$, the drag epoch precedes last scattering $z_{d}>z_{*}$ assuming standard recombination. If the universe is reionized after recombination to some ionization level $x_{e}, z_{d}=263\left(\Omega_{0} h^{2}\right)^{1 / 5} x_{e}^{-2 / 5}\left(1-Y_{p} / 2\right)^{-2 / 5} \Theta_{2.7}^{-8 / 5}$ and the drag epoch significantly precedes last scattering in most scenarios. ${ }^{5}$ Here $Y_{p}$ is the helium mass fraction $Y_{p} \simeq 0.23$.

By analogy to the photon case, it is useful to define the drag visibility function

$$
\mathscr{V}_{b}=\frac{a \dot{\tau}_{d} e^{-\tau_{d}}}{\int_{0}^{\eta_{0}} d \eta a \dot{\tau}_{d} e^{-\tau_{d}}}
$$

suitably normalized to have unity area. Diffusion damping modifies the acoustic visibility function as

$$
\hat{\mathscr{V}}_{b}=\mathscr{V}_{b} e^{-\left[k / k_{D}(\eta)\right]^{2}} \text {. }
$$

Thus, we expect that immediately after the drag epoch the baryon velocity and density perturbations are approximately

$$
V_{b}\left(\eta_{d}, k\right)=\hat{\Theta}_{1}\left(\eta_{d}, k\right) \mathscr{D}_{b}(k), \quad \delta_{b}\left(\eta_{d}, k\right)=\left[\hat{\delta}_{b}\left(\eta_{d}, k\right)-S(0, k)\right] \mathscr{D}_{b}(k)+S(0, k),
$$

where

$$
\mathscr{D}_{b}(k)=\int_{0}^{\eta_{0}} d \eta \hat{\mathscr{V}}_{b}=\int_{0}^{\eta_{0}} d \eta \mathscr{V}_{b} e^{-\left[k / k_{D}(\eta)\right]^{2}},
$$

and recall that $\hat{\Theta}_{1}$ and $\hat{\delta}_{b}$ were given in equation $(\mathrm{B} 7)$.

\section{APPENDIX D}

\section{MATTER EVOLUTION}

After horizon crossing but before the end of the drag epoch, baryons follow the acoustic solution of equation (C11) and the CDM follows its own pressureless evolution. After the drag epoch, the baryon evolution equation (A3) is identical to the cold dark matter, and their joint evolution can be expressed in terms of fluctuations in the total nonrelativistic matter density $\delta_{m}$.

\footnotetext{
5 This differs from the treatment of HSb in which $z_{d}$ was defined to be the epoch when the perturbation joined the growing mode of pressureless linear theory. The presence of a decaying mode lowers this redshift by a factor of $\frac{3}{5}$ (see Appendix D, eq. [D19]).
} 
Thus, the solution for the time evolution of the matter fluctuations requires knowledge of both the baryon and CDM perturbations at the drag epoch. The baryonic contribution was obtained in Appendix C. Let us now evaluate the CDM contributions.

\section{D1. EXACT CDM SOLUTIONS}

The evolution of CDM fluctuations is described by equation (B10). Since the curvature or $\Lambda$ terms are negligible before the drag epoch, this equation can be rewritten in terms of the equality-normalized scale factor $y=a / a_{\mathrm{eq}}$ as

$$
\frac{d^{2}}{d y^{2}} \delta_{c}+\frac{(2+3 y)}{2 y(1+y)} \frac{d}{d y} \delta_{c}=\frac{3}{2 y(1+y)} \frac{\Omega_{c}}{\Omega_{0}} \delta_{c} .
$$

Here we have assumed that the radiation contributions to the gravitational potential have decayed to zero well after horizon crossing. In typical adiabatic models, the CDM contribution usually dominates the nonrelativistic matter. Consider first the limit of negligible baryon fraction, $\Omega_{b} / \Omega_{0} \ll 1$. In this case, the matching condition at the drag epoch becomes trivial because the baryons have no effect on the CDM. If $\Omega_{c}=\Omega_{0}$, equation (D1) has the same solution before and after the drag epoch (see Peebles 1980, eqs. [12.5] and [12.9]),

$$
U_{1}=\frac{2}{3}+y, \quad U_{2}=\frac{15}{8}(2+3 y) \ln \left[\frac{(1+y)^{1 / 2}+1}{(1+y)^{1 / 2}-1}\right]-\frac{45}{4}(1+y)^{1 / 2},
$$

before curvature or $\Lambda$ domination. Matching to the radiation-dominated solution (B12), we obtain

$$
\delta_{T}(\eta, k) \simeq \delta_{c}(\eta, k)=I_{1} \Phi(0, k)\left[\frac{3}{2} \ln \left(4 I_{2} e^{-3} \frac{a_{\mathrm{eq}}}{a_{H}}\right) U_{1}(\eta)-\frac{4}{15} U_{2}(\eta)\right],
$$

for $k \gg k_{\mathrm{eq}}$.

Let us now solve equation (D1) for the case in which the contribution of baryon is not negligible. The two independent solutions are given in exact form through Gauss's hypergeometric function $F$ by

$$
U_{i}=(1+y)^{-\alpha_{i}} F\left(\alpha_{i}, \alpha_{i}+\frac{1}{2}, 2 \alpha_{i}+\frac{1}{2} ; \frac{1}{1+y}\right),
$$

where $i=1,2$ and

$$
\alpha_{i}=\frac{1+\sqrt{1+24 \Omega_{c} / \Omega_{0}}}{4},
$$

with minus and plus signs for $i=1$ and 2, respectively. Note that $\lim _{y \rightarrow \infty} U_{i}=y^{-\alpha_{i}}$. Thus, the main effect of the baryons is to slow the power-law growth of CDM after equality.

It is easy to show that these solutions are identical to equations (D2) for $\Omega_{c}=\Omega_{0}$. They also take on elementary forms for two other special cases: $\Omega_{c}=0$,

$$
U_{1}=1, \quad U_{2}=\frac{1}{2} \ln \left[\frac{(1+y)^{1 / 2}+1}{(1+y)^{1 / 2}-1}\right]
$$

and $\Omega_{c}=\frac{1}{3} \Omega_{0}$

$$
U_{1}=(1+y)^{1 / 2}, \quad U_{2}=\frac{3}{2}(1+y)^{1 / 2} \ln \left[\frac{(1+y)^{1 / 2}+1}{(1+y)^{1 / 2}-1}\right]-3 .
$$

In order to map the solution for the radiation-dominated limit (B12) onto amplitudes of $U_{1}$ and $U_{2}$, we have to take the limit as $y \rightarrow 0$ of equation (D4). By using a linear transformation of the hypergeometric function (see, e.g., eq. 15.3.9 in Abramowitz \& Stegun 1965), we find

$$
\lim _{y \rightarrow 0} U_{i}=\frac{\Gamma\left(2 \alpha_{i}+1 / 2\right)}{\Gamma\left(\alpha_{i}\right) \Gamma\left(\alpha_{i}+1 / 2\right)}\left[-\ln y+2 \psi(1)-\psi\left(\alpha_{i}\right)-\psi\left(\alpha_{i}+\frac{1}{2}\right)\right],
$$

where $\Gamma(x)$ and $\psi(x)=\Gamma^{\prime}(x) / \Gamma(x)$ are gamma and digamma functions, respectively. Matching to the radiation-dominated solution (B12) yields

$$
\delta_{c}(\eta, k)=I_{1} \Phi(0, k)\left[A_{1} U_{1}(\eta)+A_{2} U_{2}(\eta)\right],
$$

where

$$
A_{1}=-\frac{\Gamma\left(\alpha_{1}\right) \Gamma\left(\alpha_{1}+1 / 2\right)}{\Gamma\left(2 \alpha_{1}+1 / 2\right)\left[\psi\left(\alpha_{1}\right)+\psi\left(\alpha_{1}+1 / 2\right)-\psi\left(\alpha_{2}\right)-\psi\left(\alpha_{2}+1 / 2\right)\right]}\left[\ln \left(I_{2} \frac{a_{\mathrm{eq}}}{a_{H}}\right)+2 \psi(1)-\psi\left(\alpha_{2}\right)-\psi\left(\alpha_{2}+\frac{1}{2}\right)\right] .
$$




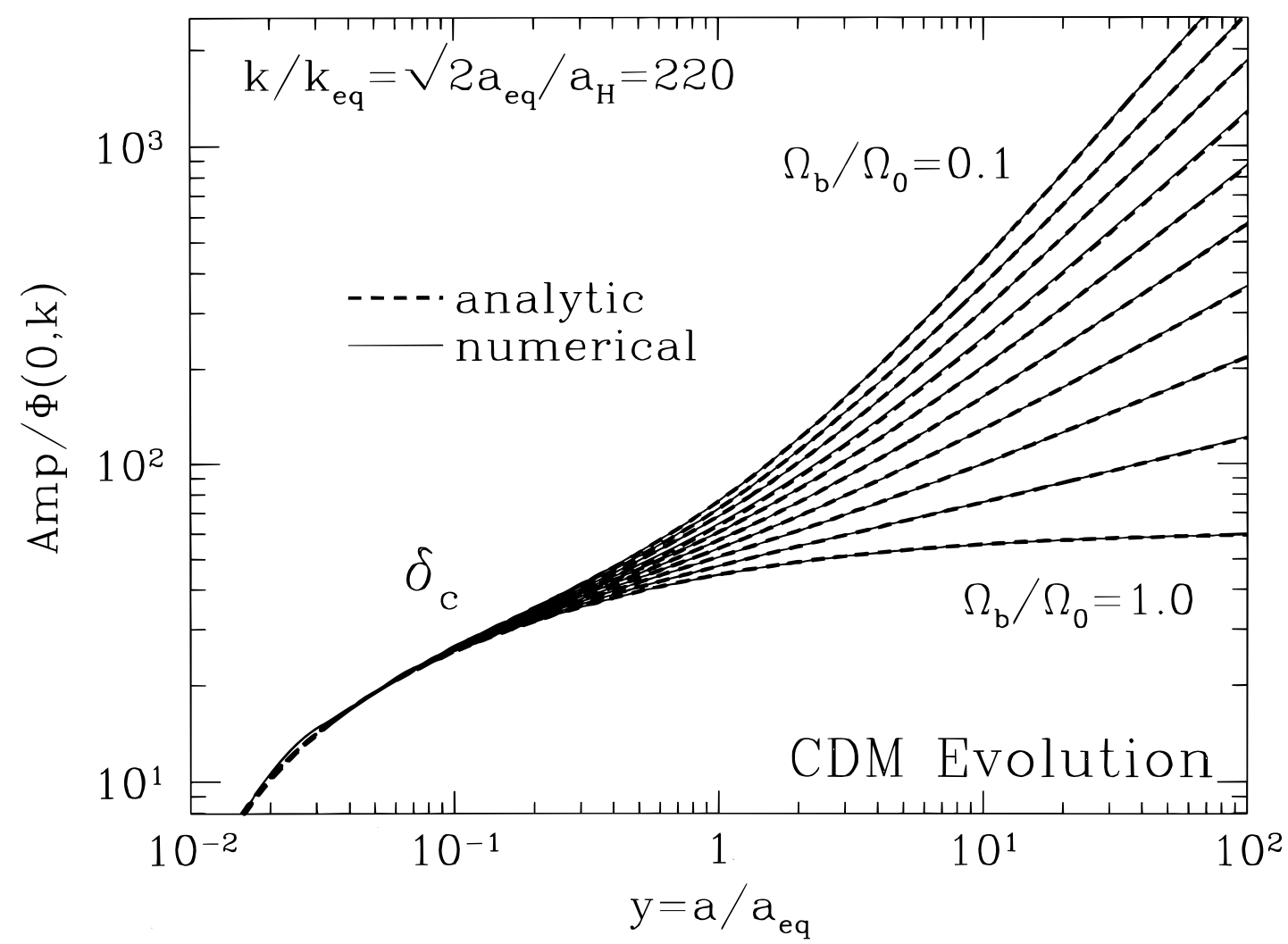

FIG. 10.-CDM evolution in the Compton drag epoch. If baryons contribute a significant fraction of the total matter density, CDM growth will be slowed between equality and the drag epoch. Held by Compton drag, the baryons do not contribute their self-gravity. For the numerical results, we choose a model that never recombined, so that $a_{d} \gg a_{\mathrm{eq}}$.

$A_{2}$ is obtained by replacing the subscripts $1 \leftrightarrow 2$ of $A_{1}$. Since $\Omega_{b} / \Omega_{0} \leq 1$, it is useful to approximate the coefficients with a series expansion,

$$
A_{1}=B_{1} \ln \left(I_{2} \frac{a_{\mathrm{eq}}}{a_{H}}\right)+B_{2}
$$

with

$$
\begin{aligned}
& B_{1}=\frac{3}{2}\left\{1-0.568\left(\Omega_{b} / \Omega_{0}\right)+0.094\left(\Omega_{b} / \Omega_{0}\right)^{2}+O\left[\left(\Omega_{b} / \Omega_{0}\right)^{3}\right]\right\}, \\
& B_{2}=\frac{3}{2}(\ln 4-3)\left\{1-1.156\left(\Omega_{b} / \Omega_{0}\right)+0.149\left(\Omega_{b} / \Omega_{0}\right)^{2}-0.074\left(\Omega_{b} / \Omega_{0}\right)^{3}+O\left[\left(\Omega_{b} / \Omega_{0}\right)^{4}\right]\right\},
\end{aligned}
$$

valid at the percent level for $\Omega_{b} / \Omega_{0}<\frac{1}{2}$, and

$$
A_{2}=-\frac{\Gamma\left(\alpha_{2}\right) \Gamma\left(\alpha_{2}+1 / 2\right)}{\Gamma\left(2 \alpha_{2}+1 / 2\right)}\left[\frac{\Gamma\left(2 \alpha_{1}+1 / 2\right)}{\Gamma\left(\alpha_{1}\right) \Gamma\left(\alpha_{1}+1 / 2\right)} A_{1}+1\right] .
$$

For the three special cases, we can describe $\delta_{c}$ by elementary functions. If $\Omega_{c}=\Omega_{0}, \delta_{c}$ is given by equation (D2), whereas

$$
\begin{aligned}
& \delta_{c}(\eta, k)=I_{1} \Phi(0, k)\left[\ln \left(4 I_{2} \frac{a_{\mathrm{eq}}}{a_{H}}\right) U_{1}-2 U_{2}\right] \quad \Omega_{c}=0, \\
& \delta_{c}(\eta, k)=I_{1} \Phi(0, k)\left[\ln \left(4 I_{2} e^{-2} \frac{a_{\mathrm{eq}}}{a_{H}}\right) U_{1}-\frac{2}{3} U_{2}\right] \quad \Omega_{c}=\frac{1}{3} \Omega_{0} .
\end{aligned}
$$

In Figure 10, we show the time evolution of $\delta_{c}$ before the drag epoch for several different values of $\Omega_{b} / \Omega_{c}$. Numerical results in this figure are for fully reionized models so that the drag epoch ends well after equality, unlike other examples in this paper.

\section{D2. MATTER TRANSFER FUNCTION}

With the baryon and CDM fluctuations at the drag epoch from equations (C11) and (D9), respectively, we can now solve for the evolution to the present. After the drag epoch, baryons behave dynamically as CDM, and the combined nonrelativistic 
matter fluctuations

$$
\begin{gathered}
\delta_{m}=\frac{\Omega_{b}}{\Omega_{0}} \delta_{b}+\left(1-\frac{\Omega_{b}}{\Omega_{0}}\right) \delta_{c}, \\
V_{m}=\frac{\Omega_{b}}{\Omega_{0}} V_{b}+\left(1-\frac{\Omega_{b}}{\Omega_{0}}\right) V_{c} .
\end{gathered}
$$

follow the growing and decaying solutions for $\delta_{m}$ (Peebles 1980),

$$
\begin{aligned}
& D_{1}=\frac{2}{3}+y, \\
& D_{2}=\frac{15}{8}(2+3 y) \ln \left[\frac{(1+y)^{1 / 2}+1}{(1+y)^{1 / 2}-1}\right]-\frac{45}{4}(1+y)^{1 / 2},
\end{aligned}
$$

before curvature or $\Lambda$ domination. To account for effects from curvature and $\Lambda$ at $a \gg a_{\mathrm{eq}}$ or $y \gg 1$, one needs simply to replace $a \rightarrow D$, where

$$
D(a)=\frac{5}{2} \Omega_{0} g(a) \int \frac{d a^{\prime}}{\left[a^{\prime} g\left(a^{\prime}\right)\right]^{3}}, \quad g^{2}(a)=a^{-3} \Omega_{0}+a^{-2}\left(1-\Omega_{0}-\Omega_{\Lambda}\right)+\Omega_{\Lambda},
$$

is the growing mode of radiationless linear theory normalized to equal $a$ at early times.

By matching the fluctuations at the drag epoch, we obtain for the growing mode

$$
\delta_{m}(\eta, k)=\left[G_{1}\left(\eta_{d}\right) \delta_{m}\left(\eta_{d}, k\right)+G_{2}\left(\eta_{d}\right) k V_{m}\left(\eta_{d}, k\right)\right] D_{1}(\eta), \quad G_{1}(\eta)=\frac{\dot{D}_{2}}{D_{1} \dot{D}_{2}-\dot{D}_{1} D_{2}}, \quad G_{2}(\eta)=\frac{D_{2}}{D_{1} \dot{D}_{2}-\dot{D}_{1} D_{2}},
$$

and similarly for the decaying mode. The combined time evolution is plotted in Figure 4 for a BDM model with $\delta_{m}=\delta_{b}$. If $z_{d} \ll z_{\text {eq }}$, equation (D18) reduces to the familiar form

$$
\delta_{m}(\eta, k)=\frac{a}{a_{d}}\left[\frac{3}{5} \delta_{m}\left(\eta_{d}, k\right)-\frac{1}{5}\left(k \eta_{d}\right) V_{m}\left(\eta_{d}, k\right)\right] .
$$

Notice that on scales much less than the horizon at the drag epoch, the velocity at $\eta_{d}$ dominates the growing mode if the two values are comparable at $\eta_{d}$ (see Fig. 11). This "velocity overshoot" effect occurs because the peculiar velocity moves the matter and creates density fluctuations kinematically. Expansion drag on the velocity eliminates it in an expansion time $\eta_{d}$, and thus causality prevents this effect from generating density fluctuation above the horizon at the drag epoch $k \eta_{d} \ll 1$.

It is conventional to recast the evolutionary effects in terms of a transfer function. As with equation (D15), we can break the present-day transfer function up into a baryonic and cold dark matter contribution at the drag epoch

$$
T(k)=\frac{\Omega_{b}}{\Omega_{0}} T_{b}(k)+\left(1-\frac{\Omega_{b}}{\Omega_{0}}\right) T_{c}(k) .
$$

It should be kept in mind that $T_{b}$ and $T_{c}$ do not represent the respective transfer functions today. Let us consider the adiabatic transfer function. Here one expresses the evolution of small-scale fluctuations in terms of those at large scales, i.e., $\left|\delta_{T}\left(\eta_{0}, k\right)\right|^{2} \propto T^{2}(k) P(k)$ with normalization $\lim _{k \rightarrow 0} T(k)=1$ and initial power spectrum $P(k) \propto\left|k^{2} \Phi(0, k)\right|^{2}$. The large-scale solution is given by (see HSa, eq. [A15])

$$
\lim _{k \rightarrow 0} \delta_{T}(\eta, k)=\left(1+\frac{4}{15} f_{v}\right)\left(1+\frac{2}{15} f_{v}\right)^{-1} \frac{6}{5}\left(\frac{k}{k_{\text {eq }}}\right)^{2} D_{1}(\eta) \Phi(0, k) .
$$

The acoustic contribution of the baryon is therefore

$$
T_{b}(k)=\left.\frac{15}{4}\left(1+\frac{4}{15} f_{v}\right)^{-1}\left(\frac{k_{\mathrm{eq}}}{k}\right)^{2} \mathscr{D}_{b}(k)(1+R)^{-1 / 4}\left(\cos k r_{s}-\frac{D_{2}}{\dot{D}_{2}} k c_{s} \sin k r_{s}\right) G_{1}\right|_{\eta=\eta_{d}} .
$$

This equation is compared with numerical results for the adiabatic transfer function in Figure $5 a$

For isocurvature BDM models, $T=T_{b}$, and it is conventional to define it such that $\left|\delta_{T}\left(\eta_{0}, k\right)\right|^{2} \propto T^{2}(k)|S(0, k)|^{2}$ with normalization $\lim _{k \rightarrow \infty} T(k)=1$. From equation (B6), the small-scale tail of the transfer function becomes

$$
T(k)=1-\left.\frac{3 \sqrt{6}}{4}\left(1-\frac{4}{15} f_{v}\right) \frac{k_{\mathrm{eq}}}{k} \mathscr{D}_{b}(k)(1+R)^{-1 / 4}\left(\sin k r_{s}-\frac{D_{2}}{\dot{D}_{2}} k c_{s} \cos k r_{s}\right)\right|_{\eta=\eta_{d}} .
$$

This function is plotted in Figure $5 b$.

In the $\Omega_{b} / \Omega_{0} \rightarrow 0$ limit, the CDM contributions can be expressed in terms of elementary functions as

$$
\lim _{\Omega_{b} \rightarrow 0} T(k)=I_{1}\left(1+\frac{2}{5} f_{v}\right)\left(1+\frac{4}{15} f_{v}\right)^{-1} \frac{5}{4}\left(\frac{k_{\mathrm{eq}}}{k}\right)^{2} \ln \left(4 I_{2} e^{-3} \frac{a_{\mathrm{eq}}}{a_{\mathrm{H}}}\right) \simeq \frac{\ln 1.8 q}{14.2 q^{2}} \quad f_{v}=0.405,
$$




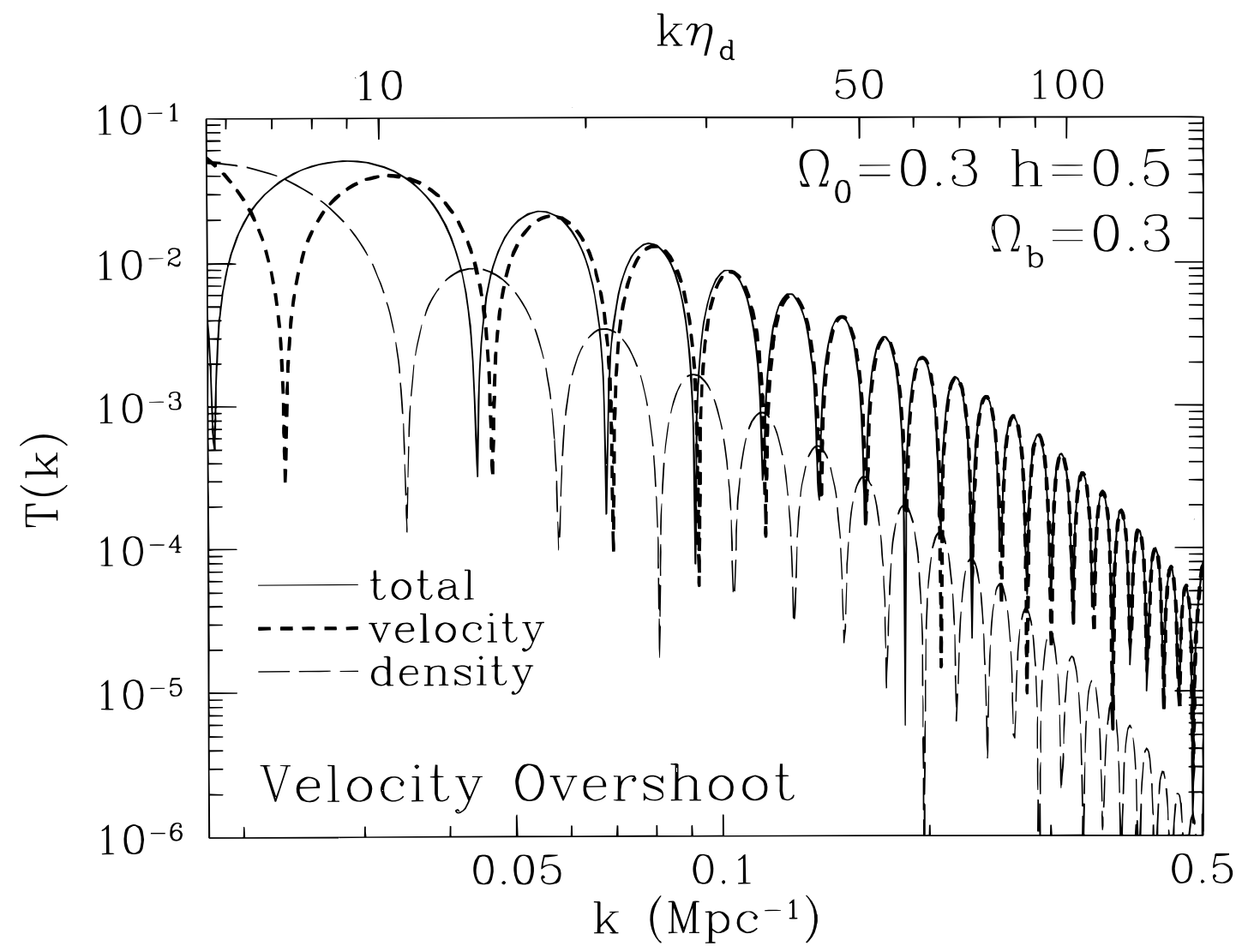

Fig. 11.-Velocity overshoot effect. Below the horizon at the drag epoch $k \eta_{d} \gg 1$, the acoustic velocity at $z_{d}$ dominates the growing mode and hence the final transfer function. Near the horizon, the acoustic density becomes comparable and shifts the zero points of the oscillation.

where $q=\left(k / \mathrm{Mpc}^{-1}\right) \Theta_{2.7}^{2}\left(\Omega_{0} h^{2}\right)^{-1}$. This should be compared with the high- $k$ tail of the standard fitting function to the numerical results (BBKS),

$$
T_{\mathrm{BBKS}}(q)=\frac{\ln (1+2.34 q)}{2.34 q}\left[1+3.89 q+(16.1 q)^{2}+(5.46 q)^{3}+(6.71 q)^{4}\right]^{-1 / 4},
$$

i.e., $\lim _{q \rightarrow \infty} T(q)=\ln (2.34 q) / 15.7 q^{2}$, which differs by $\sim 10 \%$ from the analytic prediction at small scales. In fact, since the fitting formula was designed to fit intermediate scales, equation (D24) is more accurate at extremely small scales.

If the baryon fraction is nonnegligible, the contribution is expressed in terms of hypergeometric functions through equation (D4),

$$
T_{c}=\left.I_{1}\left(1+\frac{2}{5} f_{v}\right)\left(1+\frac{4}{15} f_{v}\right)^{-1} \frac{5}{6}\left(\frac{k_{\mathrm{eq}}}{k}\right)^{2}\left[G_{1}\left(A_{1} U_{1}+A_{2} U_{2}\right)-G_{2}\left(A_{1} \dot{U}_{1}+A_{2} \dot{U}_{2}\right)\right]\right|_{\eta=\eta_{d}} .
$$

Though exact, this expression is rather complicated. It is useful and instructive to seek a simple scaling relation for this form. Notice that for all cases the scale dependence of the transfer function may be written as

$$
T_{c} \simeq \alpha \frac{\ln 1.8 \beta q}{14.2 q^{2}},
$$

for $f_{v}=0.405$. Here $\alpha$ and $\beta$ are functions of $\Omega_{0} h^{2}$ and $\Omega_{b} / \Omega_{0}$. Note that as $q \rightarrow \infty$ the modification due to $\beta$ becomes insignificant. A very accurate fit to both $\alpha$ and $\beta$ is given in Appendix E, equations (E12) and (E13). The numerical, analytic, and fitted analytic results are compared with the empirical scalings of Peacock \& Dodds (1994) and Sugiyama (1995) in Figure 12. The analytic calculation is essentially exact while the fitted analytic form works to $1 \%$ accuracy. Notice that in this extreme case we have improved significantly upon previous results.

Equation (D27) breaks down for intermediate to large scales. The CDM contribution can be scaled approximately from the BBKS form as

$$
T_{c}(k) \simeq T_{\mathrm{BBKS}}(\tilde{q}), \quad \bar{q}(k)=\frac{k}{\mathrm{Mpc}^{-1}} \alpha^{-1 / 2}\left(\Omega_{0} h^{2}\right)^{-1} \Theta_{2.7}^{2} .
$$

This expression is employed in Figure 5 with the coefficient 6.71 in equation (D25) replaced by $6.71(14.2 / 15.7)=6.07$ to match the analytic small-scale tail. Notice that at the largest scales, this expression underestimates the matter transfer function. This 


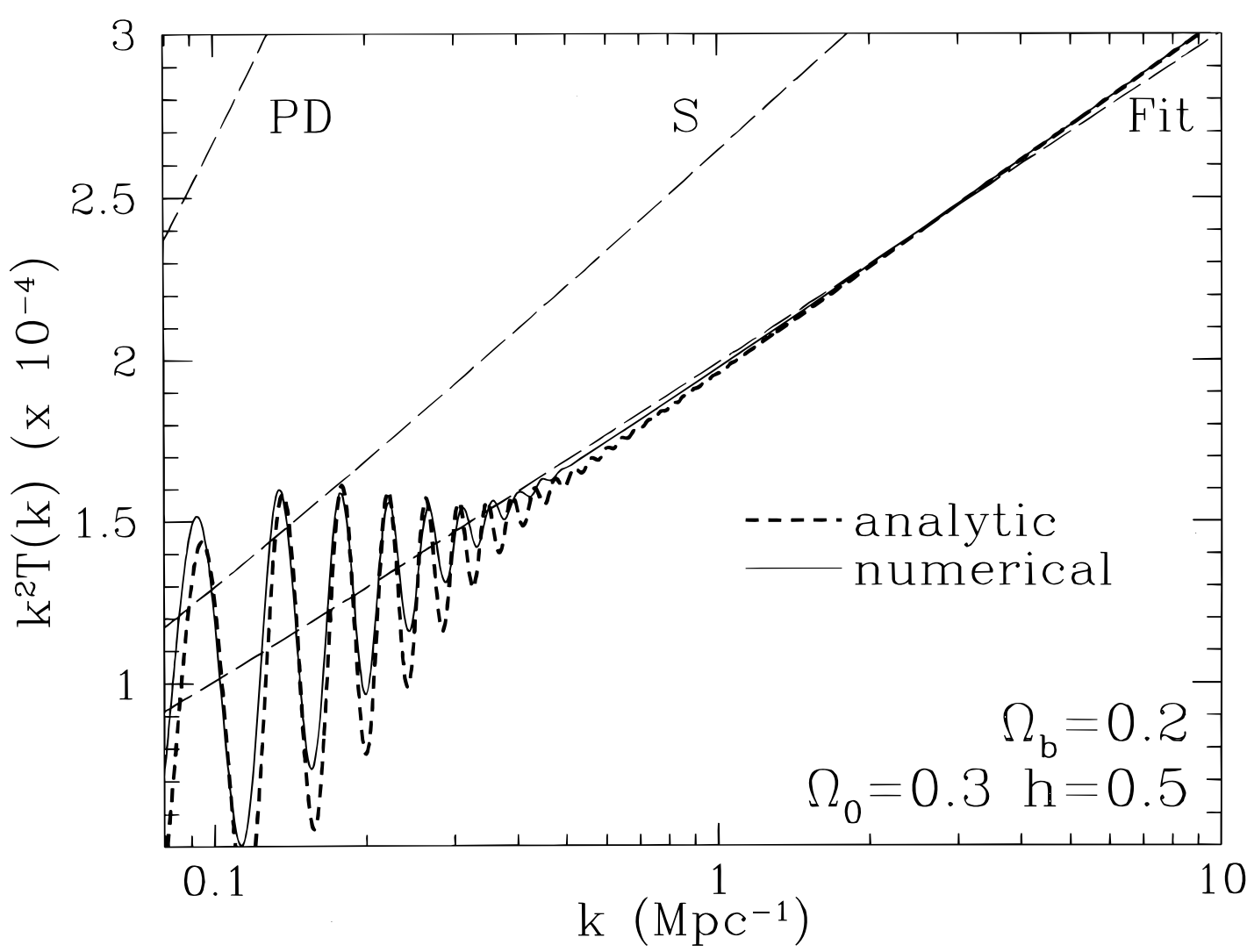

FIG. 12.-Adiabatic CDM transfer function in a high $\Omega_{b} / \Omega_{0}=\frac{2}{3}$ case. The analytic solution is essentially exact in the small-scale limit. Simple fits based on the BBKS form can cause large errors at the small scale (Peacock \& Dodds 1994; Sugiyama 1995). The fitting function developed here (see equation [D27]) works at the $1 \%$ level, even for this extreme case.

is because baryon contributions must be included properly. Although the limiting form $\lim _{k \rightarrow 0} T_{b}=1$ is simple, the behavior near the horizon scale at $z_{d}$ is not. Since this region is not the main focus of this work, we do not attempt to describe this analytically. If the baryonic oscillations are small or smoothed over, an approximate patch is given by

$$
T(k) \simeq T_{\mathrm{BBKS}}(\hat{q}), \quad \hat{q}(k)=\frac{k}{\mathrm{Mpc}^{-1}}\left(1-\frac{\Omega_{b}}{\Omega_{0}}\right)^{-1 / 2} \alpha^{-1 / 2}\left(\Omega_{0} h^{2}\right)^{-1} \Theta_{2.7}^{2},
$$

which extends the Peacock \& Dodds (1994) approach to high $\Omega_{b} / \Omega_{0}$.

Finally, we can express the ratio of the acoustic peak heights to the CDM tail with equations (D20), (D22), and (D24),

$$
\frac{\Omega_{b}}{\Omega_{c}} \frac{T_{b}}{T_{c}} \simeq \frac{\sqrt{3}}{I_{1}} k G_{2}\left(\eta_{d}\right)\left[1+R\left(\eta_{d}\right)\right]^{-3 / 4} \mathscr{D}_{b}(k)\left(1+\frac{2}{5} f_{v}\right)^{-1}\left[\ln \left(4 I_{2} e^{-3} \frac{a_{\mathrm{eq}}}{a_{\mathrm{H}}}\right)\right]^{-1} \frac{\lim _{\Omega_{b} \rightarrow 0} T_{c}}{T_{c}},
$$

if the velocity overshoot effect dominates the acoustic contributions. We can simplify this expression by noting that $G_{2}\left(\eta_{d}\right) \simeq$ $\frac{2}{5}\left(\Omega_{0} H_{0}^{2} / a_{\mathrm{eq}}\right)^{-1 / 2}\left(1+a_{d} / a_{\mathrm{eq}}\right)^{-1 / 2}$. Furthermore, the function $k \mathscr{D}_{b}$ peaks at roughly $0.8 k_{\mathrm{S}}$ with an amplitude of $0.4 k_{\mathrm{S}}$. With the scaling of equation (D27), the peak relative amplitude of the acoustic oscillation is approximately

$$
70 \frac{\Omega_{b}}{\Omega_{c}} \frac{k_{\mathrm{S}}}{\mathrm{Mpc}^{-1}} \frac{a_{\mathrm{eq}}}{\left(a_{\mathrm{eq}}+a_{d}\right)^{1 / 2}}\left[1+R\left(\eta_{d}\right)\right]^{-3 / 4}\left(\Omega_{0} h^{2}\right)^{-1 / 2} \alpha^{-1}\left[\ln \left(\frac{1.8 \beta}{\Omega_{0} h^{2}} \frac{k_{\mathrm{S}}}{\mathrm{Mpc}^{-1}}\right)\right]^{-1} .
$$

The logarithmic term is roughly unity and may be dropped for estimation purposes (see eq. [9]). Equation (D31) roughly quantifies the prominence of the acoustic oscillations in a CDM model. For best accuracy, however, the solutions (D22) and (D26) for the baryons and CDM respectively should be employed.

\section{APPENDIX E}

\section{RECOMBINATION FITTING FORMULAE}

Rather than recalculate the atomic physics of recombination each time one needs to consider effects at the last scattering and drag epochs, it is convenient to have accurate fitting formulae that incorporate the ionization history. In general, all quantities associated with the ionization history must be functions of $\Omega_{0} h^{2}$ and $\Omega_{b} h^{2}$ alone once the CMB temperature $T_{0}=2.726 \mathrm{~K}$ (Mather et al. 1994), neutrino fraction $f_{v}=0.405$, and helium fraction $Y_{p} \simeq 0.23$ are fixed. Fitting functions in 
this Appendix are designed to be valid at the percent level for an extended range of parameter space, $0.0025 \lessgtr \Omega_{b} h^{2} \lesssim 0.25$ and $0.025 \lesssim \Omega_{0} h^{2} \lesssim 0.64$, and consequently they appear rather complicated. We employ a recombination calculation based on the improvements discussed in $\mathrm{Hu}$ et al. (1995).

The last scattering epoch is a very weak function of parameters and is given by

$$
\begin{aligned}
& z_{*}=1048\left[1+0.00124\left(\Omega_{b} h^{2}\right)^{-0.738}\right]\left[1+g_{1}\left(\Omega_{0} h^{2}\right)^{g_{2}}\right], \\
& g_{1}=0.0783\left(\Omega_{b} h^{2}\right)^{-0.238}\left[1+39.5\left(\Omega_{b} h^{2}\right)^{0.763}\right]^{-1}, \\
& g_{2}=0.560\left[1+21.1\left(\Omega_{b} h^{2}\right)^{1.81}\right]^{-1} .
\end{aligned}
$$

The drag epoch ends at a related redshift that depends somewhat more strongly on the parameters

$$
\begin{aligned}
& z_{d}=1345 \frac{\left(\Omega_{0} h^{2}\right)^{0.251}}{1+0.659\left(\Omega_{0} h^{2}\right)^{0.828}}\left[1+b_{1}\left(\Omega_{b} h^{2}\right)^{b_{2}}\right], \\
& b_{1}=0.313\left(\Omega_{0} h^{2}\right)^{-0.419}\left[1+0.607\left(\Omega_{0} h^{2}\right)^{0.674}\right], \\
& b_{2}=0.238\left(\Omega_{0} h^{2}\right)^{0.223} .
\end{aligned}
$$

The two are approximately equal if $\Omega_{b} h^{2} \simeq 0.03$.

The diffusion damping envelope can be approximated through the first decade of damping by the form

$$
\mathscr{D}_{\gamma}(k) \simeq e^{-\left(k / k_{D}\right)^{m \gamma}},
$$

where the effective damping scale $k_{D \gamma}$ has simple asymptotic scaling,

$$
\frac{k_{D \gamma}}{\mathrm{Mpc}^{-1}}= \begin{cases}F_{1} & \Omega_{b} h^{2} \gg 0.1, \\ \left(\Omega_{b} h^{2}\right)^{p_{1}} F_{2} & \Omega_{b} h^{2} \ll 0.1,\end{cases}
$$

with

$$
\begin{aligned}
& F_{1}=0.293\left(\Omega_{0} h^{2}\right)^{0.545}\left[1+\left(25.1 \Omega_{0} h^{2}\right)^{-0.648}\right], \\
& F_{2}=0.524\left(\Omega_{0} h^{2}\right)^{0.505}\left[1+\left(10.5 \Omega_{0} h^{2}\right)^{-0.564}\right], \\
& p_{1}=0.29 .
\end{aligned}
$$

The basic scaling in the low $\Omega_{b} h^{2}$ limit can be understood by the Saha approximation in which the ionization fraction scales approximately as $x_{e} \propto\left(\Omega_{b} h^{2}\right)^{-1 / 2}$. Thus, the diffusion length $\lambda_{D} \sim k_{D \gamma}^{-1} \sim\left(\eta_{*} / \dot{\tau}\right)^{1 / 2} \propto \eta_{*}^{1 / 2}\left(\Omega_{b} h^{2}\right)^{-1 / 4}$. Since $\eta_{*} \propto\left(\Omega_{0} h^{2}\right)^{-1 / 2}$ in the matter-dominated high $\Omega_{0} h^{2}$ limit, this is approximately of the same form as equation (E5). For high $\Omega_{b} h^{2}$, the corrections from an accurate treatment of the atomic levels become more important due to the high Ly $\alpha$ opacity. These two simple limits can be joined accurately by a rather artificial looking but highly accurate form,

$$
\begin{aligned}
\frac{k_{D \gamma}}{\mathrm{Mpc}^{-1}} & =\left\{\frac{2}{\pi} \arctan \left[\frac{\pi}{2}\left(\frac{F_{2}}{F_{1}}\right)^{p_{2} / p_{1}}\left(\Omega_{b} h^{2}\right)^{p_{2}}\right]\right\}^{p_{1} / p_{2}} F_{1}, \\
p_{2} & =2.38\left(\Omega_{0} h^{2}\right)^{0.184} .
\end{aligned}
$$

From Figure 2, we see that this overestimates the true damping as $\Omega_{b} h^{2} \rightarrow 0$ due to a breakdown of tight coupling. It is interesting to note that the full numerical results suggest that the Saha estimation of $p_{1} \simeq 0.25$ in equations (E5) and (E6) is a somewhat better phenomenological fit.

The steepness index $m_{\gamma}$ of the diffusion damping envelope is a very weak function of cosmological parameters. In the limit that last scattering occurred instantaneously, $m_{\gamma} \rightarrow 2$. The finite width of the visibility function modifies this as

$$
m_{\gamma}=1.46\left(\Omega_{0} h^{2}\right)^{0.0303}\left(1+0.128 \arctan \left\{\ln \left[\left(32.8 \Omega_{b} h^{2}\right)^{-0.643}\right]\right\}\right),
$$

which only varies by $\sim 10 \%$ across the full range of parameter space.

Silk damping for the baryons can likewise be approximated by

$$
\mathscr{D}_{b}(k) \simeq e^{-(k / k s)^{m_{s}}},
$$

with

$$
\frac{k_{\mathrm{S}}}{\mathrm{Mpc}^{-1}}=1.38\left(\Omega_{0} h^{2}\right)^{0.398}\left(\Omega_{b} h^{2}\right)^{0.487} \frac{1+\left(96.2 \Omega_{0} h^{2}\right)^{-0.684}}{1+\left(346 \Omega_{b} h^{2}\right)^{-0.842}},
$$

and the steepness index by

$$
m_{\mathrm{S}}=1.40 \frac{\left(\Omega_{b} h^{2}\right)^{-0.0297}\left(\Omega_{0} h^{2}\right)^{0.0282}}{1+\left(781 \Omega_{b} h^{2}\right)^{-0.926}} .
$$

As is the case with the photons, the latter accounts for the width of the visibility function and is almost independent of cosmological parameters. 
TABLE 1

COMMONLY UsED SYMBoLs ${ }^{\mathrm{a}}$

\begin{tabular}{|c|c|c|}
\hline Symbol & Definition & Equation \\
\hline$\ldots$ & CMB temperature perturbation & (A1) \\
\hline$\Theta_{0}$ & CMB monopole perturbation & (A1) \\
\hline$\Theta_{1}$ & $V_{\gamma}$-CMB dipole perturbation & (A1) \\
\hline$\Pi_{T}$. & Anisotropic stress perturbation & (A5) \\
\hline & Curvature perturbation & (A5) \\
\hline ..... & Newtonian potential & (A5) \\
\hline$\Omega_{i} \ldots \ldots \ldots$ & Critical fraction in $i$ & (1) \\
\hline$\alpha \ldots \ldots \ldots \ldots$ & Baryon $T$-suppression & (E11) \\
\hline$\alpha_{1} \alpha_{2} \ldots \ldots$ & $U_{1} U_{2}$ power law & (D5) \\
\hline ..... & Baryon $T$-log correction & (E11) \\
\hline$\eta\left(\eta_{t}\right)$ & Conformal time (at $t$ ) & (A1) \\
\hline$\delta_{i} \ldots \ldots \ldots \ldots$ & Density perturbation in $i$ & (8) \\
\hline ..... & Compton optical depth & (A1) \\
\hline$\tau_{d} \ldots \ldots \ldots \ldots$ & Drag optical depth & (C8) \\
\hline$\omega \ldots \ldots \ldots \ldots$ & Acoustic frequency & (A13) \\
\hline $\mathscr{D}_{\gamma} \ldots$ & Photon damping factor & (C5) \\
\hline ..... & Baryon damping factor & (C12) \\
\hline ..... & Compton visibility & (C1) \\
\hline$\ldots$. & CMB acoustic visibility & (C3) \\
\hline .... & Drag visibility & (C9) \\
\hline ..... & Baryon acoustic visibility & (C11) \\
\hline$A_{1} A_{2} \ldots \ldots$ & Boost CDM matching condition & (D10) \\
\hline$C_{A} \ldots \ldots \ldots$ & Adiabatic acoustic amplitude & (B5) \\
\hline$C_{I} \ldots$ & Isocurvature acoustic amplitude & (B6) \\
\hline$D \ldots$ & Radiationless growth & (D17) \\
\hline$D_{1} D_{2} \ldots \ldots$ & Matter-radiation growth & (D16) \\
\hline$G_{1} G_{2} \ldots \ldots$ & Drag matching condition & (D18) \\
\hline$I_{1} I_{2} \ldots \ldots \ldots$ & CDM boost integrals & (B14) \\
\hline$U_{1} U_{2} \ldots \ldots$ & Drag CDM growth & (D4) \\
\hline$R\left(R_{t}\right) \ldots \ldots$ & $b / \gamma$ momentum (at $t$ ) & (A2) \\
\hline$T \ldots \ldots \ldots \ldots$ & Transfer function & (D20) \\
\hline$\ldots$ & Baryon $T$ drag contribution & (D22), (D23) \\
\hline ....... & CDM $T$ drag contribution & (D28) \\
\hline$V_{i} \ldots \ldots \ldots \ldots$ & Velocity in $i$ & (A1) \\
\hline$a\left(a_{t}\right) \ldots \ldots$ & Scale factor (at $t$ ) & (A1) \\
\hline$a_{H} \ldots \ldots \ldots$ & Horizon crossing $a$ & (B13) \\
\hline$c_{s} \ldots$ & Photon-baryon sound speed & (A2) \\
\hline$f_{v} \ldots$ & Neutrino fraction & (B2) \\
\hline$k \ldots$ & Wavenumber (Laplace eigenvalue) & (A1) \\
\hline$k_{D} \ldots \ldots \ldots$ & Diffusion wavenumber & (A14) \\
\hline$k_{D \gamma}$. & CMB damping wavenumber & (A14), (E6) \\
\hline$k_{\mathrm{S}} \ldots \ldots \ldots \ldots$ & Silk damping wavenumber & (6), (E9) \\
\hline$k_{\mathrm{eq}} \ldots \ldots \ldots$ & Equality horizon wavenumber & (B2) \\
\hline$k_{\gamma j} \ldots \ldots \ldots$ & $j$ th photon peak wavenumber & (2) \\
\hline$k_{b j} \ldots \ldots \ldots$ & $j$ th baryon peak wavenumber & (5) \\
\hline$m_{\gamma} \ldots \ldots \ldots \ldots$ & CMB damping steepness & (4), (E7) \\
\hline$m_{\mathrm{S}} \ldots \ldots \ldots$ & Baryon damping steepness & (6), (E10) \\
\hline$q \ldots \ldots \ldots \ldots$ & Scaled wavenumber & (D25) \\
\hline$r_{s} \ldots \ldots \ldots \ldots$ & Sound horizon & (A2) \\
\hline$y \ldots \ldots \ldots$ & $a / a_{\mathrm{eq}}$ & (D1) \\
\hline$z_{*} \ldots$ & Last scattering redshift & (E1) \\
\hline$z_{d}^{*} \ldots \ldots \ldots \ldots$ & Drag redshift & (E2) \\
\hline$z_{\mathrm{eq}} \ldots \ldots \ldots \ldots$ & Equality redshift & (1) \\
\hline
\end{tabular}

${ }^{a}$ Fluid elements are $i=b, c, \gamma, v, m, r$ for the baryons, CDM photons, neutrinos, total nonrelativistic matter, and total relativistic matter, respectively. Special epochs include $t=*, d$, eq, $H$ for last scattering, the drag epoch, matter-radiation equality, and horizon crossing, respectively. Overdots represent conformal time derivatives.

Finally, by employing equation (E2) for the drag epoch, the cumbersome analytic result for the CDM drag contribution to the small-scale transfer function $T=\left(\Omega_{b} / \Omega_{0}\right) T_{b}+\left(\Omega_{c} / \Omega_{0}\right) T_{c}$ from equation (D26) can be fitted as

$$
T_{c} \simeq \alpha \frac{\ln (1.8 \beta q)}{14.2 q^{2}},
$$

with

$$
\begin{aligned}
\alpha & =a_{1}^{-\Omega_{b} / \Omega_{0}} a_{2}^{-\left(\Omega_{b} / \Omega_{0}\right)^{3}}, \\
a_{1} & =\left(46.9 \Omega_{0} h^{2}\right)^{0.670}\left[1+\left(32.1 \Omega_{0} h^{2}\right)^{-0.532}\right], \\
a_{2} & =\left(12.0 \Omega_{0} h^{2}\right)^{0.424}\left[1+\left(45.0 \Omega_{0} h^{2}\right)^{-0.582}\right],
\end{aligned}
$$


as the suppression factor and

$$
\begin{aligned}
\beta^{-1} & =1+b_{1}\left[\left(\Omega_{c} / \Omega_{0}\right)^{b_{2}}-1\right], \\
b_{1} & =0.944\left[1+\left(458 \Omega_{0} h^{2}\right)^{-0.708}\right]^{-1}, \\
b_{2} & =\left(0.395 \Omega_{0} h^{2}\right)^{-0.0266},
\end{aligned}
$$

as the correction to the logarithm. A table of these and other commonly used symbols is provided in Table 1 .

\section{REFERENCES}

Abramowitz, M., \& Stegun, I. A. 1965, Handbook of Mathematical Functions (New York: Dover)

Bardeen, J. M., Bond, J. R., Kaiser, N., \& Szalay, A. S. 1986, ApJ, 304, 15 (BBKS)

Bond, J. R. 1996, in Cosmology and Large Scale Structure, ed. R. Schaeffer (Amsterdam: Elsevier), in press

Bond, J. R., \& Efstathiou, G. 1984, ApJ, 285, L45

Chibisov, G. V. 1972, AZh, 49, 72

Doroshkevich, A. G., Zeldovich, Ya. B., \& Sunyaev, R. A. 1978, Soviet Astron., 22, 523

Gnedin, N. Y., \& Ostriker, J. P. 1990, ApJ, 400, 1

Hu, W., Scott, D., Sugiyama, N., \& White, M. 1995, Phys. Rev. D, 52, 5498

Hu, W., \& Sugiyama, N. 1995a, ApJ, 444, 489 (HSa)

1995b, Phys. Rev. D, 51, 2599 (HSb)

Hu, W., \& White, M. 1996, A\&A, 471, 30

.1996, ApJ, in press

Jones, B. J. T., \& Wyse, R. F. G. 1985, A\&A, 149, 144

Kaiser, N. 1983, MNRAS, 202, 1169 1984, ApJ, 282, 374
Kodama, H., \& Sasaki, M. 1986, Int. J. Mod. Phys., A1, 265

Kosowsky, A. 1996, Ann. Phys., 246, 49

Mather, J. C., et al. 1994, ApJ, 420, 439

Peacock, J. A., \& Dodds, S. J. 1994, MNRAS, 267, 1020

Peebles, P. J. E. 1968, ApJ, 153, 1

. 1980, Large Scale Structure of the Universe (Princeton: Princeton

Univ. Press) 1987a, ApJ, 315, L73 $1987 \mathrm{~b}$, Nature, 327, 210

Peebles, P. J. E., \& Yu, J. T. 1970, ApJ, 162, 815

Press, W. H., \& Vishniac, E. T. 1980, ApJ, 236, 323

Sachs, R. K., \& Wolfe, A. M. 1967, ApJ, 147, 73

Silk, J. 1968, ApJ, 151, 459

Sugiyama, N. 1995, ApJS, 100, 281

Sunyaev, R. A., \& Zeldovich, Ya. B. 1970, Ap\&SS, 7, 3

Weinberg, S. 1972, Gravitation and Cosmology (New York: Wiley)

White, S. D. M., Navarro, J. F., Evrard, A. E., \& Frenk, C. S. 1993, Nature, 366,429 